\title{
Quantitative rotating frame relaxometry methods in MRI
}

\author{
Irtiza Ali Gilani ${ }^{a, b, c *}$ and Raimo Sepponen ${ }^{d}$
}

\begin{abstract}
Macromolecular degeneration and biochemical changes in tissue can be quantified using rotating frame relaxometry in MRI. It has been shown in several studies that the rotating frame longitudinal relaxation rate constant $\left(R_{1 \rho}\right)$ and the rotating frame transverse relaxation rate constant $\left(R_{2 \rho}\right)$ are sensitive biomarkers of phenomena at the cellular level. In this comprehensive review, existing MRI methods for probing the biophysical mechanisms that affect the rotating frame relaxation rates of the tissue (i.e. $R_{1}$ and $R_{2}$ ) are presented. Long acquisition times and high radiofrequency (RF) energy deposition into tissue during the process of spin-locking in rotating frame relaxometry are the major barriers to the establishment of these relaxation contrasts at high magnetic fields. Therefore, clinical applications of $R_{1 \rho}$ and $R_{2 \rho}$ MRI using on- or off-resonance RF excitation methods remain challenging. Accordingly, this review describes the theoretical and experimental approaches to the design of hard RF pulse cluster- and adiabatic RF pulse-based excitation schemes for accurate and precise measurements of $R_{1}$ and $R_{2 \rho}$. The merits and drawbacks of different MRI acquisition strategies for quantitative relaxation rate measurement in the rotating frame regime are reviewed. In addition, this review summarizes current clinical applications of rotating frame MRI sequences. Copyright @ 2016 John Wiley \& Sons, Ltd.
\end{abstract}

Keywords: $T_{1 \rho} \mathrm{MRI} ; T_{2 \rho} \mathrm{MRl}$; rotating frame relaxation rate mapping; spin-lock MRl; adiabatic pulses for $T_{1 \rho}$ and $T_{2 \rho}$ relaxation; $\mathrm{MRI}$ sequence design; quantitative relaxometry in MRl; endogenous contrast methods

\section{INTRODUCTION}

Rotating frame relaxometry was introduced into the field of MRI in 1985 (1). In MRI, the process by which the longitudinal component of the magnetization vector regains its equilibrium value under the influence of a radiofrequency (RF) field is called spin-lattice relaxation in the rotating frame of reference and is characterized by the time constant $T_{1}$. The MRI method described by Sepponen et al. (1) demonstrated the measurement of the relaxation time constant $T_{1 \rho}$ at a very low static magnetic field strength when the RF pulse was applied on-resonance to the water peak of the spectrum. Since its inception, this imaging technique has shown potential for the elucidation of physiochemical phenomena in biological tissue that occur at low interaction frequencies, i.e. approximately in the range $0.1 \mathrm{kHz}$ to a

* Correspondence to: I. A. Gilani, Brain Research Unit, Department of Neuroscience and Biomedical Engineering, Aalto University, PO Box 15100, 00076 Aalto, Finland.

E-mail: irtizagilani@yahoo.com

\section{a I. A. Gilani}

Brain Research Unit, Department of Neuroscience and Biomedical Engineering, Aalto University, Aalto, Finland

b I. A. Gilani

Advanced Magnetic Imaging Center, Aalto University, Aalto, Finland

C I. A. Gilani

National Magnetic Resonance Research Center (UMRAM), Bilkent University, Ankara, Turkey

d R. Sepponen

Department of Electronics, School of Electrical Engineering, Aalto University, Aalto, Finland few kilohertz. Several methods for the magnetization excitation, image acquisition and analysis of the rotating frame longitudinal and transverse relaxation rates, $R_{1 \rho}\left(1 / T_{1 \rho}\right)$ and $R_{2 \rho}\left(1 / T_{2 \rho}\right)$, respectively, have been employed in MRI. Advances in MRI equipment, such as high-field technology, improved methods for RF irradiation, faster gradients and state-of-the-art data acquisition techniques, have allowed more accurate and precise rotating frame relaxometry in $\mathrm{MRI}$, and these developments have enabled numerous applications that were envisaged in the past. This imaging technique has been used for rotating frame relaxation

Abbreviations used: $A F P$, adiabatic full passage; $A H P$, adiabatic half passage; $b$-FFE, balanced fast field echo; $b$-GRE, balanced gradient echo steadystate; BOLD, blood oxygenation level-dependent; BPP, Bloembergen, Purcell and Pound; b-SSFP, balanced steady-state free precession; BWR, BlochWangsness-Redfield; CESL, chemical exchange imaging with spin-lock; CEST, chemical exchange saturation transfer; CESTrho, chemical exchange saturation transfer in rotating frame; CP, Carr-Purcell; CSA, chemical shift anisotropy; CSF, cerebrospinal fluid; CW, continuous wave; EPI, echo planar imaging; FISP, fast imaging with steady-state precession; FLASH, fast low-angle shot; FIESTA, fast imaging employing steady-state acquisition; FSE, fast spin echo; Gd-DTPA, gadopentetic acid; GOIA, gradient offset-independent adiabaticity; GOIA-W, gradient offset-independent adiabaticity with WURST modulation; GRASS, gradient recalled acquisition using steady states; HS, Silver-Hoult hyperbolic secant pulse; LASER, localization by adiabatic selective refocusing; MAPSS, magnetization-prepared angle-modulated partitioned $k$-space spoiled gradient echo snapshots; MLEV, Malcolm Levitt's composite pulse decoupling; MS, multi-slice; MT, magnetization transfer; MTR asym, magnetization transfer ratio asymmetry; RAFF, relaxation along a fictitious field; RAFFn, relaxation along a fictitious field in nth rotating frame; $R F$, radiofrequency; $S A R$, specific absorption rate; SL, spin-lock/spin-locking; SLE, stochastic Liouville equation; SISSFP, spin-lock steady-state free precession; SNR, signal-to-noise ratio; $S L R$, Shinnar-Le Roux; SPGR, spoiled gradient echo; SS, single-slice; $T_{1}-F F E, T_{1^{-}}$ weighted fast field echo; TIDE, transition into driven equilibrium; True-FISP, true fast imaging with steady-state precession; TSE, turbo spin echo; TSL, spin-locking time period; UTE, ultrashort TE; WURST, wideband, uniform rate, smooth truncation. 
rate measurements of gray and white matter (2), paramagnetic substances and human gliomas (3), and in vitro and in vivo breast lesions (4); for the quantification of blood $T_{1 \rho}$ in vitro; for the determination of the effects of dissolved oxygen on $T_{1 \rho}$ in protein phantoms; for assessing the acidification of intracellular $\mathrm{pH}$ using hypercapnia in rats; and for the manipulation of tissue oxygen tension via hypoxia in rats (5), etc. As a result of its great ability to target the low-frequency spectrum, this MRI technique is promising for the analysis of progression in diseases such as ischemia (6), cartilage degradation (7), Alzheimer's disease (8), Parkinson's disease (9) and liver fibrosis (10), and for other clinical applications, i.e. meniscal $T_{1 \rho}$ measurement (11), gene therapy (12), spin-lock flow tagging for MR angiography (13), muscle imaging $(14,15)$ and differentiation of hemangiomas from liver metastases $(16,17)$, etc. It is important to mention that the technique employed in ref. (2) is termed rotating frame off-resonance $\left(T_{1 \rho \text {-off }}\right)$ imaging because the RF pulse is applied off-resonance to the water peak of the spectrum. Quantitative $R_{1 \rho}$ and $R_{2 \rho}$ relaxation maps reflect the biochemical composition of healthy and diseased tissues. These relaxation maps can be obtained by performing curve fitting using the mathematical expressions that describe the corresponding relaxation phenomena as a function of the RF pulse duration, with the amplitude of the pulse fixed in the range of a few kilohertz at low main magnetic fields $\left(B_{0}\right.$ in the range of approximately $0.1-1 \mathrm{~T})$. However, the range of RF amplitudes increases at high magnetic fields $\left(B_{0}>1.5 \mathrm{~T}\right)$, where the specific absorption rate (SAR) becomes significant.

To comprehend the theoretical and experimental concepts of rotating frame relaxometry in MRI effectively, this review article is divided into five major sections. First, an introduction to the physical processes that can be probed by this type of relaxation is provided. Second, the biophysical bases of rotating frame relaxation are described. Third, the perspectives of quantum physics employed for the modeling of rotating frame relaxation, which have been used in the literature to derive the mathematical expressions of the relaxation phenomena, are overviewed. Fourth, the RF irradiation schemes that enable spin-locking (SL) in rotating frame MRI are described. Fifth, the MRI pulse sequences that have been proposed in the literature for rotating frame MRI, some relevant applications and significant results are summarized.

\section{PROCESSES AND TIME-SCALES}

The important molecular motion phenomena that can be probed using MR are nuclear spin relaxation, diffusion and exchange processes, over a wide range of time-scales and between sites with different resonance parameters. The time-scales for motional processes, such as flow, diffusion, chemical exchange, mechanical motion, molecular rotations and molecular vibrations, are summarized in Fig. 1. Rotating frame relaxation is sensitive to the molecular processes that occur close to the effective precessional frequency on the order of kilohertz. The effective precessional frequency is influenced by the effective magnetic field, named $B_{\text {eff }}$ (explained later in the section on 'RF fields in the rotating reference frame'). Therefore, the $T_{1 \rho}$ and $T_{2 \rho}$ relaxation time constants are sensitive to slow molecular motional processes in comparison with the $T_{1}$ and $T_{2}$ relaxation time constants, and are very useful for producing MRI contrasts in vivo, which can provide insight into important biological processes that are characterized by slow molecular motions.

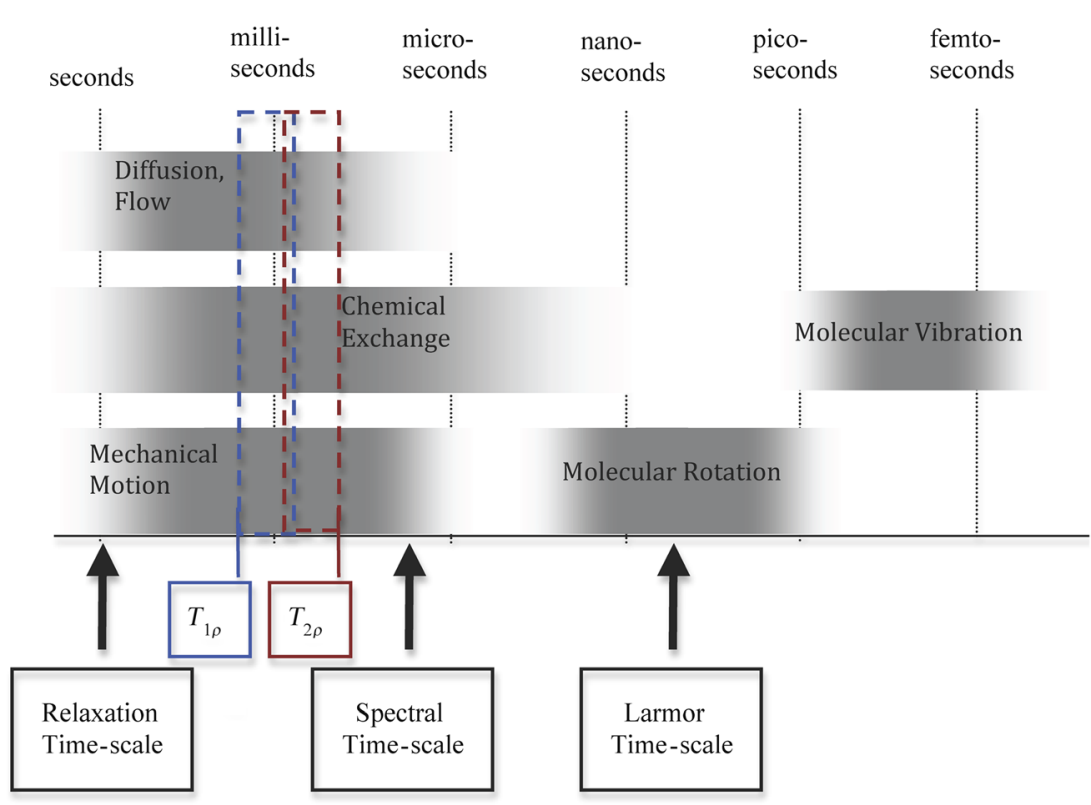

Figure 1. Typical motional time-scales for physical processes in a rotating frame MRI experiment. The time-scales of the rotating frame relaxation time constants, $T_{1 \rho}$ and $T_{2 \rho}$, are marked in blue and red, respectively. The Larmor time-scale refers to the time required for the spins to precess through 1 rad in the magnetic field. The spectral time-scale represents the time corresponding to the inverse width of the NMR spectrum, measured in frequency units. The relaxation time-scale corresponds to the value of the spin-lattice relaxation time constant. All of these time-scales depend on sample, spin isotope, magnetic field strength and temperature. Processes between the relaxation time-scale and the spectral time-scale influence the exchange dynamics of the spin population. Processes on the spectral time-scale affect the lineshapes. Processes on the Larmor time-scale are responsible for the spin-lattice relaxation. 


\section{RELAXATION MECHANISMS}

\section{Mechanisms for relaxation in rotating reference frame in MRI}

Under the influence of the applied SL field $\left(B_{1}\right)$ in rotating frame $M R I$, the relaxation phenomena occur in the low-frequency region of the spectrum. The $B_{\text {eff }}$ field rotates at a particular angle to $B_{0}$ with the Larmor frequency of the nuclei. In terms of classical vector-based terminology, the time constant for the recovery of the magnetization vector to the equilibrium value along the vector representing $B_{\text {eff }}$ is called the spin-lattice or longitudinal relaxation time constant in the rotating frame and is denoted by $T_{1 \rho}$ (or $T_{1 \rho \text {-off }}$ in the case of off-resonance irradiation). In the rotating frame, the cumulative field experienced by a spin in any state is enhanced if the magnetic fields of the surrounding spins are directed along the $B_{\text {eff }}$ field, which results in the rapid precession of the spins with the coherent phases following the RF pulse. As time progresses, the augmentative phase coherence among the spins is lost. According to the classical vector-based approach, this process can be depicted by the decay of the net transverse component of magnetization along $B_{\text {eff }}$ and the corresponding decay time constant $T_{2 \rho}$ (or $T_{2 \rho \text {-off }}$ in the case of offresonance irradiation), which is called the spin-spin relaxation time constant in the rotating frame of reference.

In the context of rotating frame nuclear spin relaxation, the variation of the local magnetic fields (occurring as a result of internal spin dynamics under the influence of the $B_{1}$ or $B_{\text {eff }}$ field) experienced by the spins in the system is the main mechanism of relaxation. The dipole-dipole interaction is influenced by the distance between the pair of spins and by their orientations relative to the $B_{1}$ or $B_{\text {eff }}$ field. Another mechanism of relaxation exists when the electronic environment around the spin is nonspherical (anisotropic). The magnitude of the electronic shielding around the spin is dependent on the molecular orientation relative to the $B_{1}$ or $B_{\text {eff }}$ field, which results in chemical shift anisotropy (CSA). CSA is another source of local fields that result from the molecular electron currents induced by the $B_{1}$ or $B_{\text {eff }}$ field. Furthermore, these local fields are modulated by molecular tumbling. An additional mechanism is the electrostatic interaction that occurs between a nucleus with an electric quadrupole moment and the electric field gradient that exists at the nuclear site as a result of the surrounding charges.

For spin $1 / 2$ nuclei, $T_{1 \rho}$ relaxation occurs in response to the fluctuating local magnetic fields at the sites of nuclear spins as a result of thermal motion or molecular tumbling. The direct dipole-dipole coupling between spins is affected by molecular tumbling, which contributes to this type of relaxation. The motion of the molecule exposes each spin to a changing magnetic field as a result of its neighboring molecule. The total magnetic field, which is the combined effect of the $B_{1}$ or $B_{\text {eff }}$ field and the local fields, is time dependent and orientation dependent, and molecular reorientation or tumbling can then modulate the corresponding spin interaction energies to cause relaxation under the influence of RF. The thermal motion of a nucleus can result in fluctuating electrostatic interaction energies, and these fluctuations produce transitions between the spin states during the rotating frame relaxation in a similar manner to dipoledipole interactions. The range of the local field fluctuations, their spatial and temporal magnitude, and the rate of spin diffusion through locally induced field gradients caused by susceptibility differences affect these relaxation rates. Another major contributor to rotating frame relaxation is chemical exchange between the groups of spins, which depends on several factors, i.e. chemical shift, temperature and exchange rate of the exchanging spins. In a heterogeneous medium, cross-relaxation in the rotating frame may admeasure exchanging spins that are affected by non-exchanging protons via through-space dipolar coupling (18). In a medium that has bulk susceptibility differences and varying chemical exchange between spins, the rotating frame relaxation rates reflect the integrated effects of diffusion, chemical exchange and molecular motion. Under appropriate diffusion conditions, the magnetic susceptibility gradient fields produce effects, such as chemical exchange and diffusion, at multiple time-scales and contribute to $T_{1 \rho}$ relaxation (19).

\section{Tissue compartmentalization}

Soft tissues are heterogeneous media that contain proteins, DNA, RNA, carbohydrates and lipids and, in addition, have circulation and extracellular matrix. The Bloembergen, Purcell and Pound (BPP) theory (20) explains the proton relaxation characteristics of solids and most monomolecular solvents (e.g. water, ethanol, glycerol, oils), but is not applicable for complex heterogeneous environments, e.g. human tissue and complex protein solutions. The water content of soft tissue in the human body is between $60 \%$ and $80 \%$. For most tissues, the relaxation characteristics are defined by the water-macromolecule interaction behavior. Complex protein systems contain minute solute structures that rotate at rapid rates, depending on the hydrodynamic characteristics (mass of water/mass of solute) of the specific solute (21). Protons are abundant in some of the solutes present in tissues and are therefore a source of the relaxation phenomena in MRI. In multicomponent macromolecular solutions composed

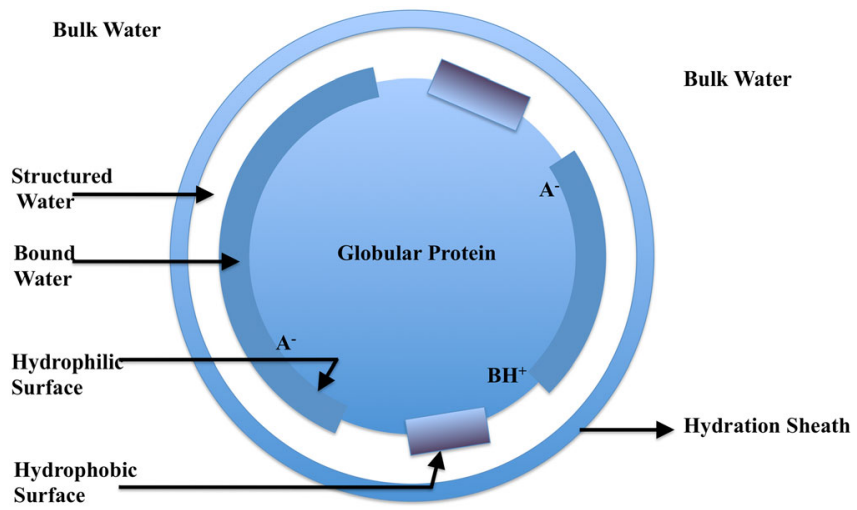

Bulk Water

Bulk Water

Figure 2. A schematic representation of bound, structured and bulk water compartments on a globular protein in a dilute solution. Basic $\left(\mathrm{BH}^{+}\right)$and acidic $\left(\mathrm{A}^{-}\right)$protein side chains interact with hydration water to form charged hydrophilic sites. Hydrophobic sites are also shown. Hydrophilic solutes are soluble in water and have either electric dipoles or fixed charges on their molecular surface that can hydrogen bond with water molecules at the sites termed hydrophilic or hydrogen-bonding sites. Hydrophobic solutes do not have hydrogen-bonding sites and they are admissible to the solution by the kind of bonding called hydrophobic bonding, which is the consequence of splitting of water-water bonds to accept a hydrophobic molecule as a neighbor to a water molecule at the sites called hydrophobic sites. Relaxation of water protons in protein solutions and in tissue is dominated by interactions at particular proteinwater interfacial sites that cover less than $1 \%$ of the protein-water interface. These are sites that hold solvent water molecules in the first hydration layer for $1 \mu \mathrm{s}$, apparently by four hydrogen bonds. 
mostly of water and macromolecules, some compartments are formed that contain protons in different molecules. These compartments exchange energy in different ways that are determined by the solute-solvent interactions in the solution. Fig. 2 illustrates these compartments in soft tissues, using a globular protein as an example.

Water molecules, which are immobilized on the surface of macromolecules with a correlation time equivalent to ice (the correlation time for chemical exchange of water is essentially the period of time a water molecule remains bonded to its four neighboring water molecules), experience rapid exchange with free bulk water molecules in solution (22). The spin-lattice relaxation rate of tissue can be expressed in terms of the rapid exchange between three water compartments, i.e. bound, structured and bulk water $(23,24)$. Water molecules that are directly hydrogen bonded to the electric dipole site or the ionic charge site on the macromolecule (with typically two or more ligands) are classified as bound water. There is an ion-bound domain that contains water molecules bound to ionic charge sites. Similarly, there is a polar-bound domain that contains water molecules bound to polar (electric dipole) sites. Bound water is composed of both ion-bound and polar-bound domains. Water molecules that are motionally perturbed by a macromolecule, but not bonded to it, are termed structured water. All bound or structured water molecules are collectively called hydration water. Bulk water contains water molecules that are distant from the macromolecule in the region in which molecular motion is determined by the intrinsic interaction characteristics of the water molecule itself.

The geometric organization of the polar sites and non-polar sites on the macromolecule influences the compartmentalization process of water. Non-polar regions of the macromolecule influence the rotation of water molecules in their vicinity in an anisotropic fashion to establish hydrogen bonds with the neighboring water molecules. Water molecules near the polar sites form hydrogen bonds with the macromolecules through the polar sites. Structured water is aligned with the bound water section whilst suffering hydrophobic motional restrictions in the case of nearby non-polar sites (21).

\section{Effects of tissue compartments on the relaxation in the rotating reference frame}

Because the solid content of most tissue types (excluding those with significant lipid content) is predominantly protein, it may be reasonable to consider protein solution for any tissue relaxation model (solute protein is immobilized). Variations in the ability of different tissues to perturb the intrinsic motion of the water molecules (formation of bound and structured water compartments) on or near their molecular surfaces are related to the protein content and composition, and the polymerization state of the proteins. The rotating frame relaxation behavior at a specific resonance frequency depends on the relative contributions of the different tissue compartments, which is related to molecular motion and other phenomena relative to the frequency selected by the strength of the $B_{1}$ field. The relaxation behavior of the macromolecular compartment is depicted in Fig. 3, in which the internal macromolecular motion dominates the relaxation characteristics at Larmor resonance frequency and chemical exchange and diffusion have a more profound influence at the low resonance frequencies usually employed for rotating frame relaxometry in MRI. The bound and free water compartment

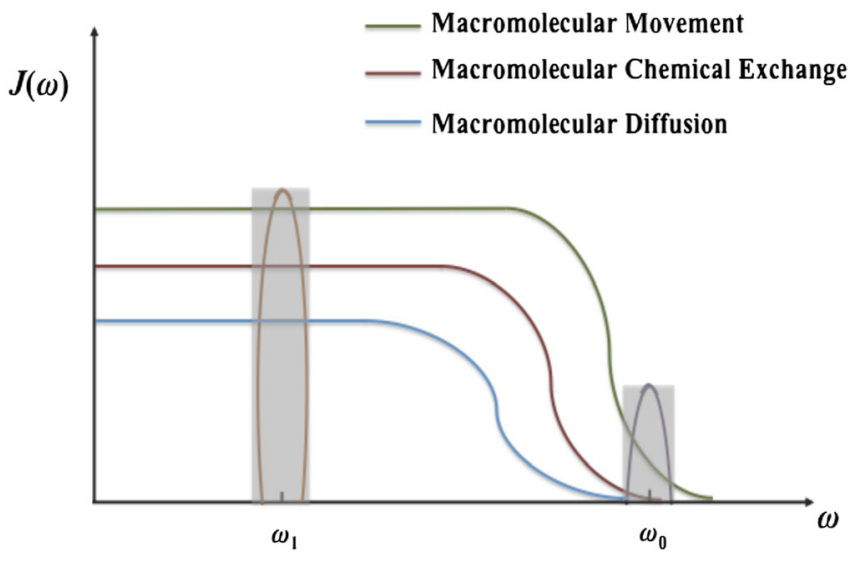

Figure 3. The spectral distribution $J(\omega)$ represents the density of the nuclei rotating at each frequency in the macromolecular environment. The spectral distribution is plotted versus the frequency of nuclei contributing to distribution by motion in the macromolecular environment (green line), the frequency of nuclei contributing to distribution by chemical exchange (red line) and the frequency of nuclei contributing to distribution by diffusion (blue line). The frequency $\omega_{0}$ is the normal resonance frequency in the MRI experiment and the frequency $\omega_{1}$ represents the resonance frequency in the rotating frame experiment. The shaded area around the rotating frame resonance frequency represents the contribution of the different nuclei to the cumulative spectral distribution with a correlation time that matches the resonance frequency. It should be noted in the shaded region around $\omega_{0}$ that the nuclei contributing by diffusion and chemical exchange are less dense or negligible, and only the nuclei contributing to the spectral density by motion are shown. Alternatively, around $\omega_{1}$ in the rotating frame MRI, the dense cumulative spectral distribution of the macromolecular movement, chemical exchange diffusion of the nuclei can be probed.

contributions to relaxation are illustrated in Fig. 4. Fig. 4 shows that, at the Larmor frequency, motion and other relaxation mechanisms of the free water compartment are dominant, because the same mechanisms of the macromolecular compartment and the bound water compartment are not the most effective relaxation mechanisms at that frequency. In the range of the low resonance frequencies used in rotating frame relaxometry, bound water begins to contribute to the relaxation mechanism. Therefore, the spin-lattice relaxation rate of free water is longer than the macromolecular solid's rate, and the macromolecular solid's rate is longer than the bound water compartment's rate, in rotating frame relaxometry.

\section{Variants of rotating frame relaxation in MRI}

By changing the $B_{1}$ strength, variations in the rotating frame relaxation rates can be observed, and the MRI method that measures these variations is called $T_{1 \rho}$ dispersion (or $T_{1 \rho \text {-off }}$ dispersion in the case of off-resonance irradiation) imaging (25-28). This type of imaging can provide information about macromolecular size distribution, molecular motion and chemical exchange over a range of time-scales. In the context of multi-component rotating frame relaxation measurements, the values and fractions of $T_{1 \rho}$ strongly depend on both the specimen orientation and the SL field strength (29).

Typically, in the case of tissues, if the SL frequency is much lower than the spectral linewidth of the macromolecular protons, the cross-relaxation effects are reduced. These effects may be eliminated during SL imaging at the magic angle. However, at higher magnetic fields, the chemical exchange contributes 


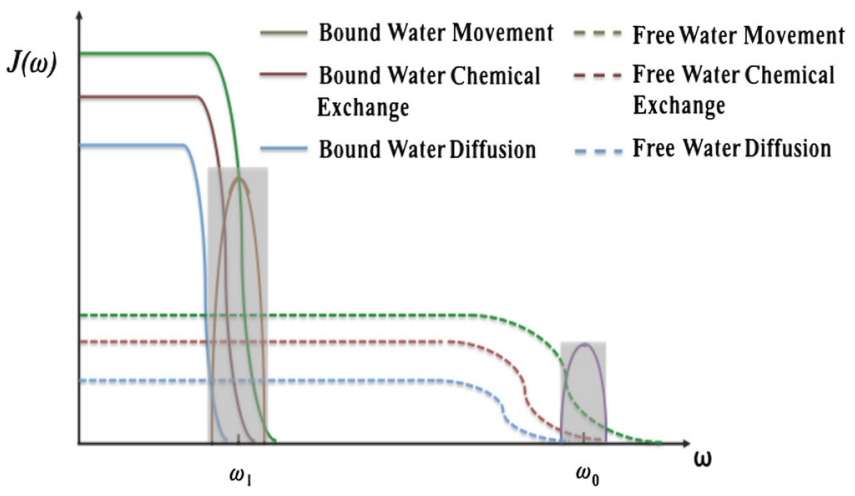

Figure 4. The spectral distribution is plotted versus the frequency for bound water nuclei and free water nuclei contributing to distribution by mechanical motion/movement, which is mentioned in Fig. 1 (full green line and broken green line, respectively), bound water nuclei and free water nuclei contributing to distribution by chemical exchange (full red line and broken red line, respectively) and bound water nuclei and free water nuclei contributing to distribution by diffusion (full blue line and broken blue line, respectively). The frequency $\omega_{0}$ is the normal resonance frequency in the MRI experiment and the frequency $\omega_{1}$ represents the resonance frequency in the rotating frame experiment. The shaded area around the rotating frame resonance frequency represents the contribution of the free water or bound water nuclei to the cumulative spectral distribution with a correlation time that matches the resonance frequency. It should be noted that the rotating frame MRI is capable of probing the spectral region that contains contributions from both bound water and free water as well as macromolecular nuclei.

significantly because of the greater resonance frequency separation between water and amide protons. There are a few theoretical models that describe the chemical exchange effects on the origins of $T_{1 \rho}$ dispersion in tissue. Usually, a two-pool model is used to describe $T_{1 \rho}$ relaxation, where the chemical exchange between water and labile protons in the solute may occur at specific sites, such as hydroxyl and amine groups. These solutes resonate at a frequency different from water and contribute an additional relaxation term to $T_{1 \rho}$ relaxation. The Chopra model (30) was proposed to determine the solvent exchange rate using different SL field strengths. This model assumes that the nuclei involved in the chemical exchange experience different chemical shifts and relaxation rates when moving between phases, which is suitable for biopolymers with properties of strong dipolar relaxation and for the range of SL frequencies used in $T_{1 \rho}$ dispersion. In the study by Cobb et al. (19), it was demonstrated that the combined chemical and fast diffusive exchange may be approximated as a fast and slow chemical exchange component in the $T_{1 \rho}$ dispersion curves that were fitted using an extension of Chopra's chemical exchange model. Therefore, $T_{1 \rho}$ dispersion imaging potentially can be used for particle size characterization or the estimation of bulk magnetic field susceptibilities in tissues with different chemical exchange and diffusion relaxation properties, such as iron deposits and lesions associated with diseases, e.g. multiple sclerosis or Alzheimer's disease. Recently, another model was introduced by Cobb et al. (18), which is based on twice differentiating the analytical expression of the Chopra model with respect to SL pulse amplitude to determine the inflection point of the $T_{1 \rho}$ dispersion curve. Consequently, an exchange rate can be estimated using a knowledge of the chemical shift of the exchanging nuclei and the rate of variation of $R_{1 \rho}$ with the SL pulse amplitude.

In a technique called magnetization transfer (MT) MRI, the saturation pulse (off-resonance preparation pulse) is applied to saturate the bound protons of tissue. In this method, the SL preparation pulse is applied at the Larmor frequency, directly saturating mobile water protons, or it can be applied closer to the resonance than the MT saturation pulse with the variable direct saturation effect of mobile water protons depending on the frequency offset, duration and amplitude of the preparation pulse (31). However, MT imaging is primarily sensitive to macromolecule-water interactions in tissues and the mobility of the solid pool. Brown and Koenig (32) proposed that, for protein-rich tissues, both MT and SL can be considered as major relaxation mechanisms. Strong correlations were found between on-resonance $T_{1 \rho}$ and MT effects (33), and between $T_{1 \rho}$ dispersion and MT effects (34), in head and neck tumors. In a phantom study by Ramadan et al. (35), the combined effect of gadopentetic acid (Gd-DTPA) and $T_{1 \rho \text {-off }}$ at small offset angles was demonstrated to be very similar to the combined effect of Gd-DTPA and MT.

Recently, in a technique called chemical exchange imaging with spin-lock (CESL) (36), theoretical analysis and numerical simulations of the $z$ spectrum and the asymmetric magnetization transfer ratio $\left(\right.$ MTR $\left._{\text {asym }}\right)$ were performed using a general twopool $T_{1 \rho}$ relaxation model beyond the fast exchange limit. Consequently, the classical analytical chemical exchange saturation transfer (CEST) model could also be derived from the $T_{1 \rho}$ relaxation model for CESL and may better characterize chemical exchange processes, from slow and intermediate to fast proton exchange rates, through the tuning of the SL pulse parameters.

\section{MATHEMATICAL EXPRESSIONS}

\section{Longitudinal and transverse relaxation in the rotating ref- erence frame (dipole-dipole interaction)}

The relaxation rate of the system of through-space dipolar coupled spins $1 / 2$ ('like' spins) can be treated as the sum of the relaxation rates of the individual spins interacting with the other spins in the ensemble. In a single spin $1 / 2$ system or a multi-spin $1 / 2$ system (formed by dipolar coupled spins in condensed matter), second-order spatial harmonics can be used to describe the spatial component of the Hamiltonians of the dipolar coupling and the molecular motions that result from the reorientations of molecules or chemical groups (37). According to BlochWangsness-Redfield (BWR) relaxation theory, the fluctuating spin Hamiltonians can be represented using the autocorrelation of stochastically stationary functions, where only the magnitude of the time interval matters rather than the absolute time (38). According to the time-dependent perturbation theory, the spin transition probability per time unit is proportional to the spectral density of the fluctuating coupling that induces the spin transition, where the spectral density is the Fourier transform of the autocorrelation function. This holds true in the case of a rotating frame of reference, and the mathematical expressions for the rotating frame relaxation rates caused by dipole-dipole interaction are derived in refs. (37-41) using the approach summarized in Fig. 5.

The rotating frame spin-lattice relaxation rate of 'like' spins directly reflects the spin transition probabilities per time unit for zero-, single- and double-quantum transitions (37), and therefore is proportional to a linear combination of spectral densities in the following form:

$$
\frac{1}{T_{1 \rho}}=\frac{3}{20 r^{6}}\left(\frac{\mu_{0}}{4 \pi}\right)^{2} r^{4}\left(3 I_{0}\left(2 \omega_{1}\right)+5 I_{1}\left(\omega_{0}\right)+2 I_{2}\left(2 \omega_{0}\right)\right)
$$




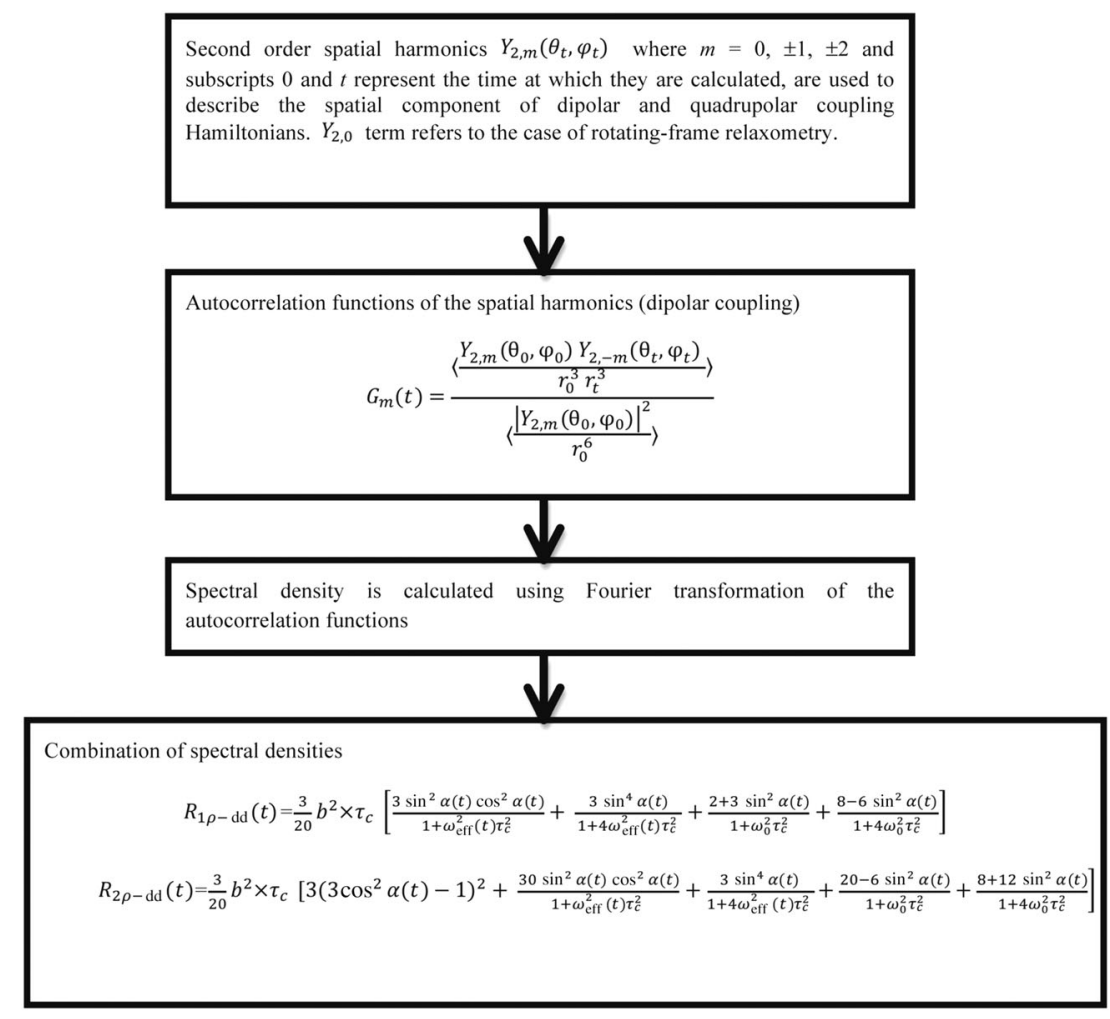

Figure 5. A flow chart depicting the process for derivation of the expressions of rotating frame relaxation rates caused by dipole-dipole interactions. Time dependence is shown for the case of adiabatic radiofrequency (RF) excitation and the same expressions apply for the case of continuous wave (CW) RF excitation, but $\omega_{\text {eff }}$ and $\alpha$ are time invariant.

where $\omega_{0}$ is the resonance frequency depending on the gyromagnetic ratio of the nuclei and the external magnetic flux density, $\omega_{1}$ is the frequency of the RF pulse employed for the rotating frame relaxometry, $I_{1}$ and $I_{2}$ are spectral densities and their subscripts 1 and 2 correspond to the $m$ of $G_{m}$ mentioned in Fig. 5. Specific constants are used to represent the dominating spin coupling type, i.e. $r^{6}$ and $\mu_{0}$. This analytical form is valid for a system of two 'like' dipolar coupled spins $1 / 2$. This expression can be modified to evaluate the time-dependent longitudinal relaxation rate $R_{1 \rho-\mathrm{dd}}(t)$. Similar expressions have been derived for the transverse relaxation in the rotating frame caused by dipolar interactions (41), which are characterized by the transverse relaxation rate $R_{2 \rho-\mathrm{dd}}(t)$, as illustrated in Fig. 5 .

\section{Longitudinal and transverse relaxation in the rotating ref- erence frame (chemical exchange)}

Under equilibrium conditions in a macromolecular solution, the chemical kinetic processes are described by chemical exchange principles. For isochronous exchange, in which two sites resonate at the same frequency, the asymptotic apparent longitudinal relaxation time constant in the rotating frame $\left(T_{1 \rho}\right)$ was measured for human occipital lobe water at $4 \mathrm{~T}$ using brief imaging readouts following an excitation pulse and a train of adiabatic full passage (AFP) pulses (frequency swept) with no interpulse intervals (42). This relaxation mechanism was modeled using a two-site exchange model for an equilibrium isochronous process whose exchange conditions were modulated during the adiabatic pulse, and a two-spin description of dipolar interaction fluctuations in each site was employed. Because $R_{1 \rho \mathrm{A}}\left(R_{1 \rho}\right.$ of macromolecule-interacting water site $\mathrm{A}$ ) was significantly modulated, whereas $R_{1 \rho \mathrm{B}}\left(R_{1 \rho}\right.$ of bulk-like water site $B)$ was not, the intrinsic relaxographic shutter speed for the process ( $\left|R_{1 \rho \mathrm{A}}-R_{1 \rho \mathrm{B}}\right|$, thus the exchange condition) was modulated. Here, $R_{1 \rho \mathrm{A}}(t)$ and $R_{1 \rho \mathrm{B}}(t)$ are the intrinsic longitudinal rotating frame relaxation rates of the sites $A$ and $B$ in the absence of exchange. The relaxation rates $R_{1 \rho \mathrm{A}}(t)$ and $R_{1 \rho \mathrm{B}}(t)$ represent relaxation from mechanisms other than the equilibrium exchange, such as dipolar interactions, cross-relaxations and crosscorrelations. If $P_{\mathrm{A}}$ and $P_{\mathrm{B}}$ represent the intrinsic equilibrium populations (mole fractions) of spins in sites $A$ and $B$, respectively, and $\hat{l}_{\mathrm{A}}$ and $\hat{l}_{\mathrm{B}}$ are the unidirectional rate constants for the site outlet (i.e. $\tau_{C A}{ }^{-1}$ and $\tau_{C B}^{-1}$, respectively) of an isothermal tissue system, their dependence on each other is given by the McConnell relationship $\left(P_{\mathrm{A}} \&_{\mathrm{A}}=P_{\mathrm{B}} \ell_{\mathrm{B}}\right)$. The rate constant, $f=l_{\mathrm{A}}+l_{\mathrm{B}}$, is the measure of the equilibrium kinetics. For isochronous two-site exchange, the apparent rotating frame relaxation rate $\left[R_{1 \rho \mathrm{A}}^{\prime}(t)\right]$ and population of the site $\mathrm{A}\left(P_{\mathrm{A}}^{\prime}\right)$ can depend on the intrinsic parameters of site $B\left[R_{1 \rho \mathrm{B}}^{\prime}(t)\right.$ and $\left.P_{\mathrm{B}}^{\prime}\right]$, and vice versa. In the fast exchange limit, the relaxation has a singlevalued rate constant $R_{1 \rho}^{\prime}(t)$ (it varies during the adiabatic rotation), i.e. $R_{1 \rho}^{\prime}(t)=P_{\mathrm{A}} R_{1 \rho \mathrm{A}}(t)+P_{\mathrm{B}} R_{1 \rho \mathrm{B}}(t)$. In the slow exchange limit, $P_{\mathrm{A}}^{\prime}=P_{\mathrm{A}}$ and $P_{\mathrm{B}}^{\prime}=P_{\mathrm{B}}$, and $R_{1 \rho \mathrm{A}}^{\prime}(t)=R_{1 \rho \mathrm{A}}(t)+k_{\mathrm{A}}, R_{1 \rho \mathrm{B}}^{\prime}(t)=R_{1 \rho \mathrm{B}}(t)+k_{\mathrm{B}}$. It is important to note that there is an $R_{1 \rho-d d}(t)$ value for each site that produces $R_{1 \rho \mathrm{A}}(t)$ and $R_{1 \rho \mathrm{B}}(t)$ values, which, together with $P_{\mathrm{A}}, P_{\mathrm{B}}, l_{\mathrm{A}}$ and $f_{\mathrm{B}}$, ultimately give $R_{1 \rho \mathrm{A}}^{\prime}(t), R_{1 \rho \mathrm{B}}^{\prime}(t), P_{\mathrm{A}}^{\prime}(t)$ and $P_{\mathrm{B}}^{\prime}(t)$, which, in turn, can give the pulse train length dependence of the signal intensity.

However, the chemical exchange phenomena between two sites in a solution that have different chemical shifts can also alternatively be quantified by the longitudinal relaxation rate, named $R_{1 \rho-\text { ex }}$. Assuming that the consequent relaxation is dominated by a single exponential damping constant, slow exchange 
regime and the population sites are unequal and asymmetric, the Bloch-McConnell equation can be used to describe the longitudinal relaxation rate constant in the rotating frame for the two-site exchange phenomena $\left(R_{1 \rho-\text { ex }}\right)$. A generalization of such expressions has been described previously using both the conventional perturbation approach for the evolution of the density operator in the interaction frame of reference (for fast exchange regimes) and the stochastic Liouville equation (SLE) for the evolution of the average density operator in the rotating frame of reference (for all exchange regimes) $(43,44)$.

In the conventional perturbation method, the elementary coupling and exchange processes are equally considered for the evaluation of the spin system evolution, which undergoes both random coupling with the lattice and chemical exchange. First, relaxation is considered in the presence of an applied RF field $B_{1}$ without chemical exchange, and relaxation is calculated for the spin operators in a tilted rotating frame in which the $z$ axis is aligned along the direction of the effective field $B_{\text {eff }}$ in the rotating frame. Subsequently, this result is extended to include the chemical exchange. The exchange rate can be categorized as slow, intermediate or fast according to the chemical shift time-scale if it is much smaller than, comparable with or much greater than the difference between the Larmor frequencies of the two sites, $\delta \omega$. Given that the two sites are exchanging with the firstorder reaction rate constant $\left(k_{0}=k_{A}+k_{B}\right)$ and the Larmor frequencies of both sites differ from the frequency of the $B_{1}$ field, the linearized expressions of $R_{1 \rho-\mathrm{ex}}$ outside the fast exchange limit and in the fast exchange limit, $\left(\left(\omega_{\text {aeff }}^{2} \omega_{\text {beff }}^{2} / \omega_{\text {eff }}^{2}\right)+k^{2} » \omega_{\text {eff }}^{2}+k^{2}\right)$, are described in a study by Trott and Palmer (45). The process of deriving the expressions for $T_{1 \rho-\mathrm{ex}}$ is outlined in Fig. 6. Four other cases were derived by Abergel and Palmer (43). In the first case, the fast exchange kinetic regime holds, where the chemical exchange rate constant is much greater than the difference between the Larmor frequencies of the exchanging spins. In the second case, one of the sites is much more populated than the other. The third case is a situation in which the frequency of the applied RF field coincides with the Larmor frequency of the population average. In the fourth case, the relaxation time constants $T_{1}$ and $T_{2}$ are equal. The time-dependent relaxation rates, $R_{1 \rho-\mathrm{ex}}(t)$ and $R_{2 \rho-\mathrm{ex}}(t)$, which were derived in refs. (46-48), are mentioned in Fig. 7.

\section{RF FIELDS IN THE ROTATING REFERENCE FRAME}

In the context of classical continuous wave SL (CW-SL) excitation, the polarizing magnetic field $B_{0}$ is slowly swept through the complete frequency range, including the resonance frequency. Alternatively, the carrier frequency of the $B_{1}$ field can also be swept. Consequently, the spins are aligned along or perpendicular to the direction of the effective field $\left(B_{\text {eff }}\right)$. The precession of the magnetization vector $M$ appears to be stopped in the rotating reference frame, which rotates at the same rate as the SL field with the frequency $\omega_{\mathrm{RF}}$. A transformation from the laboratory frame coordinates to the rotating frame coordinates can be performed by subtracting $\omega_{\mathrm{RF}}$ from the Larmor frequency $\omega_{0}$, resulting in a $z$-directed field with a value of $\omega_{0}-\omega_{\mathrm{RF}} / \gamma$. The net effective field $B_{\text {eff }}$ is then the vector sum of $\omega_{0}-\omega_{\mathrm{RF}} / \gamma$ and $B_{1}$ oriented at an angle of $\arctan \left[\left(\omega_{0}-\omega_{\mathrm{RF}} / \gamma\right) / B_{1}\right]$ degrees or radians. The amplitude of the effective frequency $\left(\omega_{\text {eff }}\right)$ is $\sqrt{ } \omega_{1}^{2}+\Delta \omega^{2}$, where $\omega_{1}$ is the amplitude of the SL pulse. However, if the $B_{1}$ field is tuned to exactly match the Larmor frequency, the net effective field is simply $B_{1}$ and the desired flip angle is generated as pure spin-tip in the $x-z$ plane by selecting a specific $B_{1}$ amplitude and duration. SL can be performed using adiabatic pulses, where the different spectral components are rotated in succession during the carrier frequency variation or frequency sweep. During an adiabatic frequency sweep, from one side of the resonance to the other, the net rotation of the magnetization vector $M$ remains highly insensitive to the changes in $B_{1}$ amplitude. The frequency sweeping feature of the adiabatic pulses can be useful for addressing the complexities associated with the spatial heterogeneity of molecular parameters. For example, variations in the exchange rates across a lesion can occur as a result of a spread in $\mathrm{pH}$ values related to disease. In the adiabatic fast passage technique, the magnetization is rotated in a short time compared with the relaxation times. During an adiabatic pulse, both amplitude and frequency are typically modulated. In the next section, the classical method to achieve SL using the typical $B_{\text {eff }}$ is described. Afterwards, adiabatic pulses for SL using $B_{\text {eff }}$ with time-varying magnitude and direction are explained.

\section{On-resonance and off-resonance SL experiment}

In a typical off-resonance SL experiment, the locked magnetization relaxes along $B_{\text {eff }}$ during the spin-locking time period (TSL). Therefore, the observed relaxation time $T_{1 \rho}$ along the direction of $B_{\text {eff }}$ probes a very low-frequency range; therefore, $T_{1 \rho}$ is likely to be more sensitive to changes in the concentration, mobility and interactions of macromolecules than $T_{1}$. In the onresonance SL technique (shown in Fig. 8), $B_{\text {eff }}$ is aligned along the $y^{\prime}$ axis. Therefore, the $T_{1 \rho}$ relaxation occurs along the $y^{\prime}$ axis. Typically, an admixture of spin-tip and SL excitation results from this process, which precesses about $B_{\text {eff }}$ in a conical structure, as shown in Fig. $8 \mathrm{~A}$. Initially, a hard $90^{\circ}$ pulse tilts the magnetization aligned with the $z$ axis on the $x-y$ plane. In the TSL period, during which the amplitude of the SL pulse is $B_{1}$, the $T_{1}$-prepared magnetization remains locked for the time of TSL and relaxes towards the previously attained equilibrium at the relaxation rate $R_{1}$. Subsequently, another hard $90^{\circ}$ pulse (with $180^{\circ}$ phase offset) tilts the relaxed $T_{1 \rho}$-prepared magnetization back to the direction of the $z$ axis. This process is illustrated in Fig. 8A, B. In Fig. $8 \mathrm{~B}$, the application of rotary echoes, which was introduced by Solomon (49), using a phase-alternating (self-compensating) $\mathrm{SL}$ pulse, is illustrated. Rotary echoes can alternate the phase of the RF pulse at the middle of the SL pulse period to refocus the $B_{1}$ inhomogeneities; rotary echoes can be combined with the spin echoes to refocus both $B_{0}$ and $B_{1}$ inhomogeneities. Witschey et al. $(50,51)$ described the methods for compensation of these artifacts. At very low locking field strengths, $T_{1 \rho}$ is approximately $T_{2}$ ( $T_{1 \rho}$ approaches $T_{2}$ in the Redfield limit). The SL excitation for the $T_{2 \rho}$-weighted imaging is illustrated in Fig. 9. To establish the $T_{2 \rho}$ contrast, all the steps are the same as for $T_{1 \rho}$ excitation, except that the phase difference of the SL pulse should be $0^{\circ}$ relative to the first $90^{\circ}$ hard pulse and its phase should be flipped by $180^{\circ}$ halfway through the SL pulse. Fig. 9 shows that the precession of the magnetization is mainly in the $y^{\prime}-z^{\prime}$ plane during the SL pulse. At the end of the SL pulse for the $T_{2 \rho}$ imaging, the magnetization is restored along the longitudinal axis by the second hard $90^{\circ}$ pulse applied along the $x$ axis with the phase of $180^{\circ}(52)$. 
Bloch-McConnell equation

Can not treat spin systems for which the chemical exchange process alters scalar coupling constants

Modified Bloch-McConnell equation

Conventional perturbation approach

Evolution of the density operator in the interaction frame of reference (limited to the fast-exchange regime)
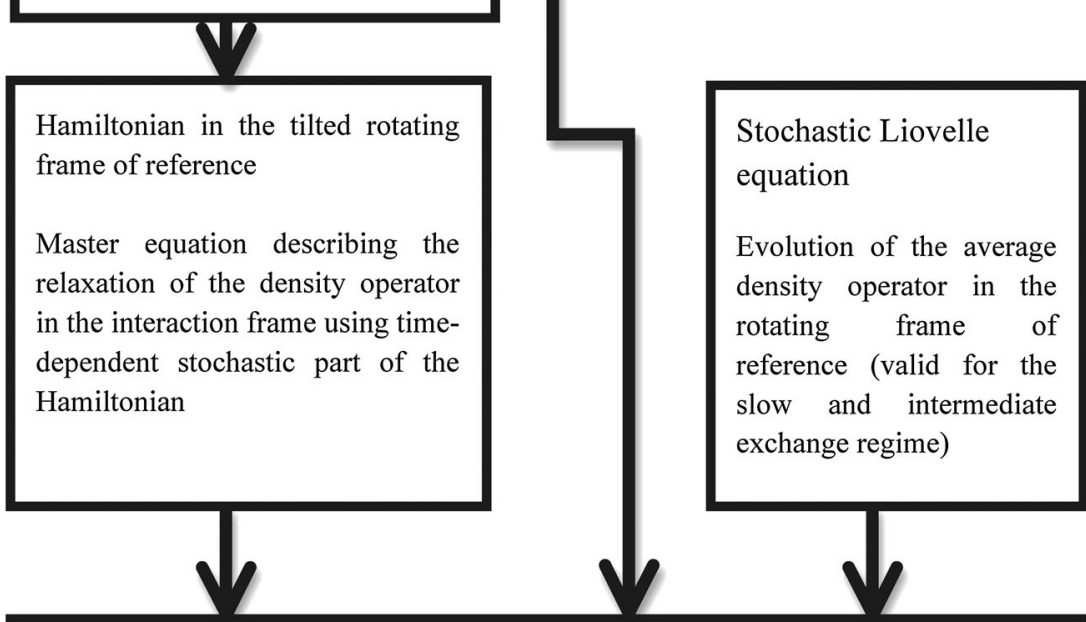

In the fast exchange limit,

$$
\begin{aligned}
& R_{1 \rho-e x}=R_{1} \cos ^{2} \theta+R_{2} \sin ^{2} \theta+\frac{\sin ^{2} \theta P_{a} P_{b} \theta^{2} k}{\omega_{e f f}^{2}+k^{2}} \\
& R_{2 \rho-e x}=R_{1} \cos ^{2} \theta+R_{2} \sin ^{2} \theta+\frac{\sin ^{2} \theta P_{a} P_{b} \theta^{2} k}{\omega_{e f f}^{2}+k^{2}}+\frac{\cos ^{2} \theta P_{a} P_{b} \theta^{2}}{k}
\end{aligned}
$$

Outside the fast exchange limit, the expression for longitudinal rotating frame relaxation rate becomes,

$$
R_{1 \rho-e x}=R_{1} \cos ^{2} \theta+R_{2} \sin ^{2} \theta+\frac{\sin ^{2} \theta P_{a} P_{b} \theta^{2} h}{\left(\omega_{\text {aeff }}^{2} \omega_{\text {beff }}^{2} / \omega_{\text {eff }}^{2}\right)+h^{2}}
$$

Figure 6. A flow chart depicting the process for derivation of the expressions of rotating frame relaxation rates caused by the chemical exchange mechanism in the case of continuous wave (CW) spin-locking (SL) excitation. $R_{1}$ and $R_{2}$ are the intrinsic longitudinal and transverse relaxation time constants, respectively, resulting from processes other than chemical exchange. $P_{\mathrm{a}}=k_{\mathrm{A}} / \ell_{\mathrm{i}}$ and $P_{\mathrm{b}}=\ell_{\mathrm{B}} / \ell_{\mathrm{o}}$ are the site populations. $\omega_{\text {aeff }}^{2}$ and $\omega_{\text {beff }}^{2}$ are the effective frequencies (sum of Larmor frequency and field inhomogeneity factor) at each site. In the fast exchange limit, $\mathrm{R}_{1 \rho \text {-ex }}$ does not depend on $\delta, P_{\mathrm{a}}$ and $P_{\mathrm{b}}$ separately, but only on their combination; therefore, it is not possible to determine them independently of each other. In contrast, in the generalized expression outside the fast exchange limit, independent determination of these quantities is possible because of the dependence of the denominator on these parameters through $\omega_{\text {aeff }}^{2}$ and $\omega_{\text {beff. }}^{2}$

In off-resonance SL excitation, an off-resonance $B_{1}$ pulse is applied, where $\omega_{0}-\omega_{\mathrm{RF}} \neq 0$. In this case, if $B_{1}$ is turned on slowly relative to the Larmor frequency, the magnetization remains

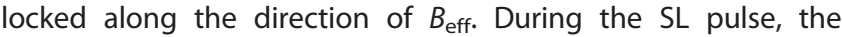

magnetization along the direction of $B_{\text {eff }}$ approaches an equilib-

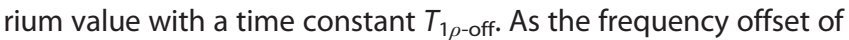
the locking field $B_{1}$ increases, the off-resonance experiment approaches the MT experiment and $T_{1}$ starts to contribute to 
Hamiltonian in the tilted rotating frame of reference

Master equation describing the relaxation of the density operator in the interaction frame using time-dependent stochastic part of the Hamiltonian

Solution of Master Equation

$$
\begin{aligned}
& R_{1 \rho-\mathrm{ex}}(t)=P_{m} P_{q} \delta \omega^{2} \sin ^{2} \alpha(t) \frac{\tau_{\mathrm{ex}}}{1+\left(\tau_{\mathrm{ex}} \omega_{\mathrm{eff}}(t)\right)^{2}} \\
& R_{2 \rho-\mathrm{ex}}(t)=P_{m} P_{q} \delta \omega^{2} \cos ^{2} \alpha(t) \tau_{\mathrm{ex}}
\end{aligned}
$$

Figure 7. A flow chart depicting the process for derivation of the expressions of rotating frame relaxation rates caused by the chemical exchange mechanism in the case of adiabatic spin-locking (SL) excitation. The correlation time for exchange phenomena is given as: $\tau_{\mathrm{ex}} \equiv 1 / \tau_{\mathrm{A}}^{-1}+\tau_{\mathrm{B}}^{-1}$. It should be noted that the time dependence of $R_{2 \rho-e x}$ arises from the time dependence of $\alpha(t)$ only.

$T_{1 \rho \text {-off, }}$ whereas the angle between $B_{\text {eff }}$ and the $z$ axis approaches zero. The relaxation time $T_{1 \rho \text {-off }}$ approaches the $T_{1 \rho}$ value when the direction of $B_{\text {eff }}$ approaches the $x^{\prime}-y^{\prime}$ plane. The transverse relaxation in the off-resonance $S L$ regime $T_{2 \rho \text {-off }}$ can be defined in a similar manner. The dependence of $T_{1 \rho \text {-off }}$ on the $B_{\text {eff }}$ strength is quantified using $T_{1 \rho \text {-off }}$ dispersion imaging (53), whereas SL field cycling relaxometry is used to measure the dependence of $T_{1 \rho \text {-off }}$ on $\omega_{\text {eff }}$ (54). $T_{1 \rho \text {-off }}$ dispersion is performed by changing the offset frequency whilst keeping $B_{1}$ constant, so that the angle between $B_{\text {eff }}$ and the $z$ axis changes. By changing this angle, the $T_{1 \rho \text {-off }}$ differences between parietal and frontal white matter were measured by Ramadan et al. (2). Their data demonstrated that $T_{1 \rho \text {-off }}$ dispersion has the potential for normal brain matter discrimination.

\section{Frames of reference for an SL experiment using adiabatic pulses}

SL can be achieved when the orientation of $B_{\text {eff }}(t)$, denoted by $\alpha(t)$, changes more slowly than the rotation of the magnetization about $B_{\text {eff }}(t)$ during an adiabatic RF passage. In addition, a uniform flip angle is attained for the spins precessing within the frequency band used for the adiabatic passage. The rotating frame relaxation mechanisms that occur during adiabatic irradiation depend on the initial orientation of the magnetization vector. $T_{1 \rho}$ relaxation occurs when the magnetization vector is aligned along the $z$ axis when the adiabatic pulse is applied and the magnetization vector remains oriented along the timedependent direction of $B_{\text {eff }}(t) . T_{2 \rho}$ relaxation occurs when the magnetization vector is in the $x^{\prime}-y^{\prime}$ plane when the adiabatic pulse is applied and the magnetization vector precesses in the plane perpendicular to $B_{\text {eff }}(t)$.

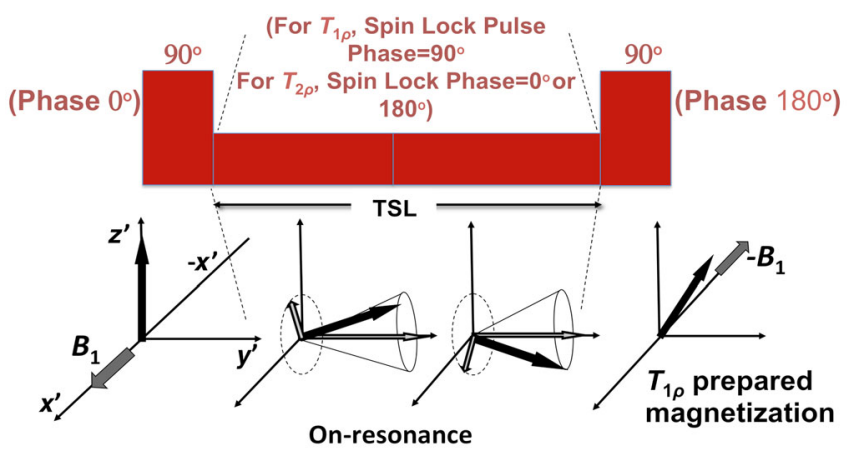

A

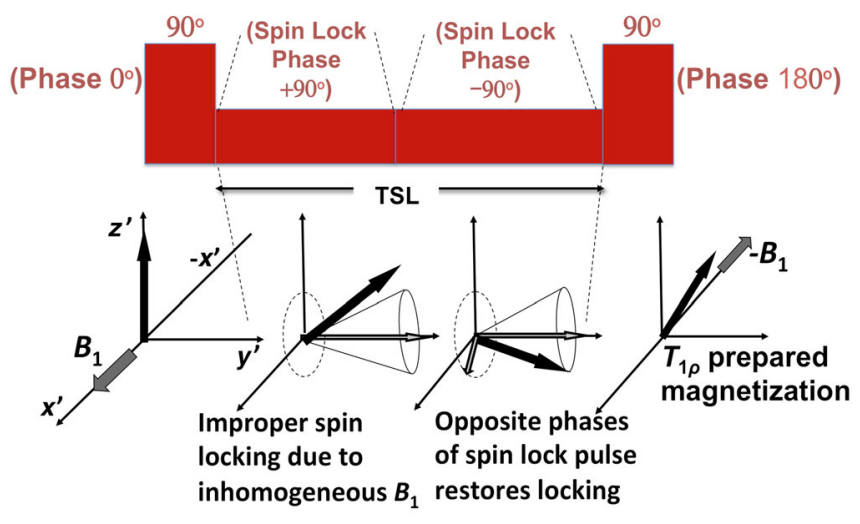

B

Figure 8. A spin-lock cluster for on-resonance $T_{1 \rho}$ imaging is shown in (A). The direction of the conical shape shows the direction of $B_{\text {eff. }}$ Spinlock and spin-tip components of the magnetization vector are shown by the non-filled arrows. These are the projections of the spin vector (shown in black) onto the axis of the cone and onto the base of the cone, respectively. The direction of the applied $B_{1}$ field is also shown. In the spin-lock cluster for on-resonance $T_{1 \rho}$ imaging, self-compensating radiofrequency (RF) pulses can be used to reduce the artifacts caused by $B_{1}$ inhomogeneity, as shown in (B).

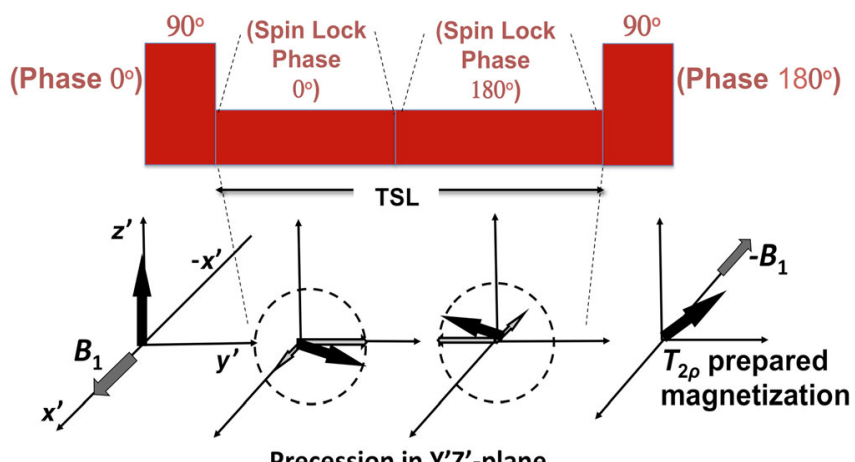

Precession in Y'Z'-plane

Figure 9. A spin-lock cluster for on-resonance $T_{2 \rho}$ imaging is shown. The precession of the locked magnetization in the $y^{\prime}-z^{\prime}$ plane is shown by the broken circle. The locked magnetization vector is shown by the filled black arrow and the corresponding components along the $y^{\prime}$ (the spinlock component) and $x^{\prime}$ (the spin-tip component) planes are shown by the non-filled arrows. In this case, the spin-tip component is much smaller than the spin-lock component and is magnified in this diagram for illustration. 
The motion of the spins induced by an adiabatic pulse can be visualized in a reference frame $x^{\prime} y^{\prime} z^{\prime}$ that rotates about the $z$ axis, collinear with $B_{0}$. This frame of reference rotates at the instantaneous frequency $\omega_{\mathrm{RF}}(t)$ of the RF pulse and is called the frequency-modulated frame, in which the RF field vector $B_{1}(t)$ does not precess in the transverse plane and remains fixed during the adiabatic period. Another frame of reference $\left[B_{\text {eff }}(t)\right.$ frame] is denoted by $x^{\prime \prime} y^{\prime \prime} z$. This second rotating frame changes its orientation with $B_{\text {eff }}(t)$, relative to the frequency modulated frame, at the instantaneous angular velocity $\mathrm{d} \alpha(t) / \mathrm{d} t$. The effective field in the second rotating frame is denoted by $B_{\text {eff }}^{\prime}(t)$ and is the vector sum of $B_{\text {eff }}(t)$ and $(\mathrm{d} \alpha(t) / \mathrm{d} t) / \gamma$ along $y^{\prime \prime}$, as shown in Fig. 10. When the adiabatic condition is well satisfied, the components of the magnetization vectors, which initially are collinear with $B_{\text {eff }}(t)$, will remain locked parallel to $B_{\text {eff }}(t)$ in the second rotating frame $x^{\prime \prime} y^{\prime \prime} z$ " during the pulse in which $B_{\text {eff }}(t)$ changes with time. Meanwhile, vectors that are perpendicular to $B_{\text {eff }}(t)$ at the onset of the pulse will remain in the plane perpendicular to $B_{\text {eff }}(t)$ and will rotate in the second rotating frame $x^{\prime \prime} y^{\prime \prime} z^{\prime \prime}$ about $B_{\text {eff }}(t)$ through an angle $\beta(t)=\gamma \int_{0}^{t} B_{\text {eff }}\left(t^{\prime}\right) \mathrm{d} t^{\prime}$.

Because the effective magnetic field is time dependent during the adiabatic pulse, the rotating frame relaxation rates are also time dependent, and therefore are a function of the pulse modulation functions, i.e. time-dependent pulse amplitude $\omega_{1}(t)$ and time-dependent pulse frequency $\omega_{\mathrm{RF}}(t)$. The amplitude, frequency modulation and time-bandwidth product values of the hyperbolic secant 1 (HS1) and hyperbolic secant 4 (HS4) pulses have been detailed by Mangia et al. (41). This study analyzes the dependences of $T_{1 \rho}$ and $T_{2 \rho}$ on the amplitude and direction of $B_{\text {eff }}(t)$ during conventional SL irradiation, as well as the pulse length, bandwidth and peak amplitude of the adiabatic pulse and the effect of different modulation functions during adiabatic irradiation.

Adiabatic half passage (AHP) and AFP pulses are the adiabatic pulses that are employed to generate uniform excitation $\left(90^{\circ}\right)$ and inversion $\left(180^{\circ}\right)$, respectively. The different stages of onresonance rotation of $B_{\text {eff }}(t)$ and magnetization during an AFP pulse can be seen in fig. 3 in the study by Tannús and Garwood (55). The pulse timings of the adiabatic irradiation schemes used in the studies by Gröhn et al. (56) and Michaeli et al. (57) are shown in Fig. 11. $T_{1 \rho}$ imaging was accomplished using an AHP pulse to generate the SL contrast (56), as illustrated in Fig. 11A. AHP was applied with a sufficiently high $B_{1}$ value to ensure an adiabatic condition, followed by $B_{1}$ ramp for the period of TSL, to reduce the amplitude of the $B_{1}$ field to the desired SL field amplitude. In a study by Michaeli et al. (42), variable numbers of HS1

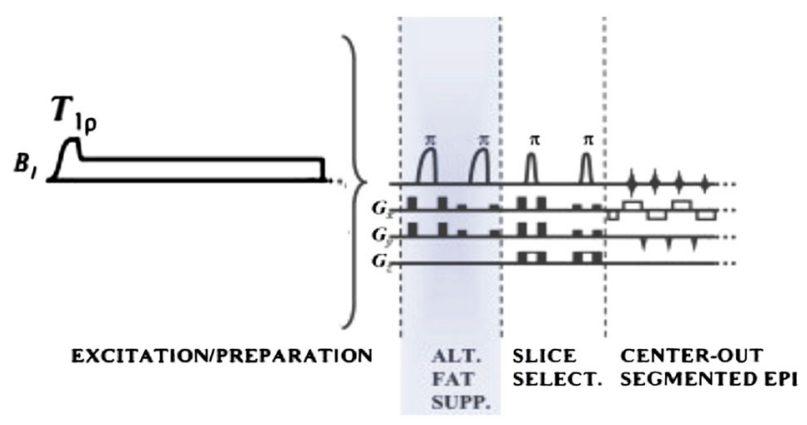

A

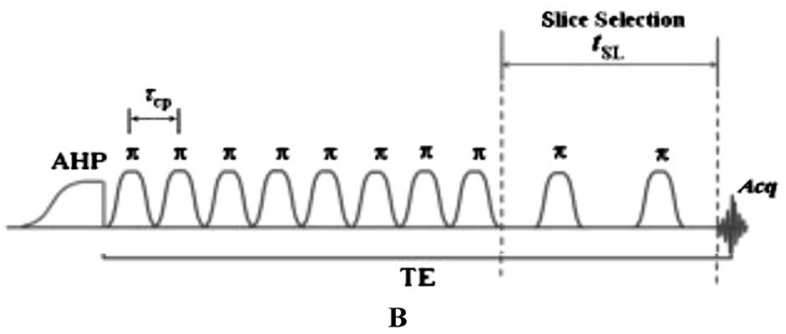

Figure 11. (A) Adiabatic irradiation scheme for $T_{1 \rho}$ imaging with adiabatic half passage (AHP) pulse. (B) Adiabatic irradiation scheme for $T_{2 \rho}$ imaging with Carr-Purcell (CP) train. [Adapted from refs. (56,57), respectively.]

or HS4 pulses were used in an AFP train, followed by coherence excitation with an AHP pulse. $T_{2 \rho}$ imaging was performed by Michaeli et al. (57) using variable numbers of HS1 or HS4 pulses in the Carr-Purcell (CP) train of the fully adiabatic pulse sequence with no time delays between adiabatic pulses, as shown in Fig. 11B. The expressions for signal intensity decay and relaxation during the entire fully adiabatic CP sequence are provided in a study by Michaeli et al. (42).

\section{Relaxation along a fictitious field}

A new method, entitled relaxation along a fictitious field (RAFF), was introduced by Liimatainen et al. (58). Relaxation dispersion was created using a fictitious field $E$ and altering its magnitude and orientation in a doubly (second) rotating frame of reference by the frequency-modulated RF pulses in the first rotating frame. This method incorporates the properties of the second rotating frame relaxation method, is sensitive to slow motion and efficiently refocuses the rotating frame rotary echo. An increase in the magnitude of the SL field was achieved without increasing

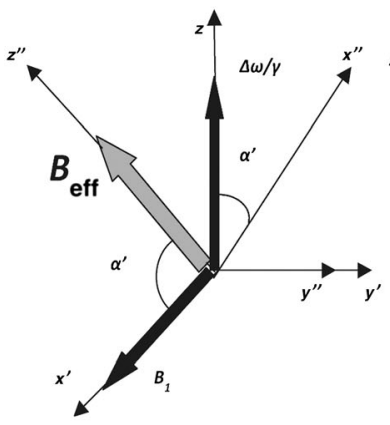

A

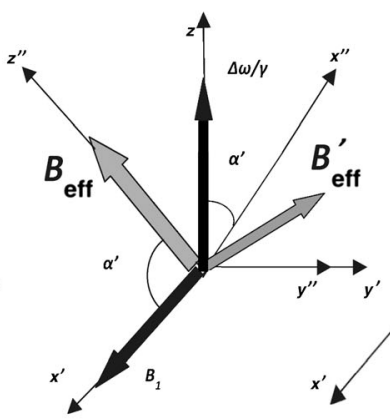

B

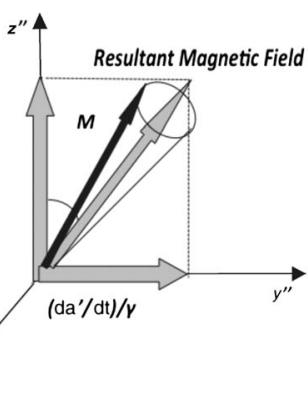

C

Figure 10. (A) $B_{\text {eff }}$ frame. (B) $B_{\text {eff }}^{\prime}$ frame. (C) Precession of the magnetization vector $\boldsymbol{M}$ around the resultant magnetic field in the doubly rotating frame. 
RF power using the fictitious field $E$ at an amplitude greater than the RF amplitude used in the transmission of RAFF. The relaxation rate during RAFF is dependent on the tilt angle of the fictitious field $E$, and is influenced by the dipolar interactions and exchange-induced relaxation between spins with different chemical shifts. The relaxation rates obtained with RAFF are slower than those with conventional off-resonance SL $T_{1}$. Therefore, this technique could potentially be used at ultrahigh fields, where the relaxations induced by dynamic averaging are accelerated and require lower RF power. It is important to mention that the RAFF method operates in the second rotating frame (rank =2) using a non-adiabatic sweep of the effective field to generate the fictitious magnetic field. Recently, in a study by Liimatainen et al. (59), the RAFF method was extended to generate MRI contrasts in the rotating frames of ranks $n>2$ (RAFFn), for example, $n=3,4,5$. The effective field in the $n$th rotating frame is the vector sum of the effective field in the $n-1$ rotating frame and a large fictitious field component, which results from sweeping the effective field in the $n-1$ rotating frame. In human and rat brains, the relaxograms depict greater separation between gray and white matter with increased $n$. RAFFn has contributions from both $T_{1 \rho}$ and $T_{2 \rho}$ in the $n$th rotating frame. In addition, RAFFn is simultaneously sensitive to dipolar interactions, diffusion and/or exchange. For adiabatic $T_{1 \rho}$, the contribution of the saturation transfer from off-resonance spins within the bandwidth of the adiabatic pulse is minor (up to 10\%) (60). However, the contribution of off-resonance saturation to RAFFn relaxation is unknown. Indeed, with increased $n$ values, the sensitivity of RAFFn to slow exchange increases, which provides a possible reason for the increased gray and white matter contrast observed with RAFF4 and RAFF5. The bandwidth of the RAFFn pulses significantly increases when $n$ increases, which is partially a result of the lower tip angle of RAFF5 compared with RAFF2. Decreased SAR, together with larger bandwidth, promise broader applications for RAFFn $(n>2)$ methods compared with the previously developed RAFF2 and SL $T_{1 \rho}$ techniques (59).

\section{PULSE SEQUENCES FOR ROTATING FRAME MRI}

There are numerous MRI pulse sequences for the establishment of rotating frame relaxation contrast. Generally, $T_{1 \rho}$ and $T_{2 \rho}$ pulse sequences are composed of two modules, i.e. the magnetization preparation module, which is used to sensitize the signal to the rotating frame relaxation phenomenon during the SL irradiation period, and the magnetization acquisition module. After magnetization preparation, the SL magnetization can be acquired using two-dimensional single-slice, multi-slice and three-dimensional pulse sequence techniques. $T_{1}$-weighted sequences are usually based on spoiled gradient echo generation [i.e. fast low-angle shot (FLASH), spoiled gradient echo (SPGR) and $T_{1}$-weighted fast field echo ( $T_{1}$-FFE) sequences] or unspoiled gradient echo generation [i.e. fast imaging with steady-state precession (FISP), gradient recalled acquisition using steady states (GRASS), FFE sequences, multiple gradient echoes in a single TR of echo planar imaging, named GE-EPI sequence, and single spin echo variations and multiple spin echo variations in a single TR, known as fast spin echo (FSE) sequence]. Three different trajectories may be used for the readout process in a sequential or interleaved manner, i.e. rectilinear, radial or spiral trajectories. Minor changes in any of the readout parameters (TE, TR, etc.) can yield significant variations in the measured $T_{1 \rho}$ or $T_{2 \rho}$. This is the major problem for consistent $T_{1 \rho}$ and $T_{2 \rho}$ quantification. To avoid discrepancies in the interpretation of the rotating frame relaxation times, it is necessary to carefully specify the readout sequence parameters, such as RF pulse type, flip angle, TE and $\mathrm{TR}$, etc. In the following sections, the characteristics of different types of sequences for $T_{1 \rho}$ or $T_{2 \rho}$ measurements are discussed.

\section{$T_{1 \rho}$ sequences and their applications}

\section{Three-dimensional gradient echo sequences}

A three-dimensional gradient echo sequence was implemented on a very low field strength using adiabatic pulses and RF spoiling (3). The major limitation of this method was the long adiabatic SL excitation scheme, which was unsuitable for the desired SAR at higher $B_{0}$ field strengths (3T and higher than 3T). Another three-dimensional $T_{1}$-weighted pulse sequence has been proposed for clinical imaging at $1.5 \mathrm{~T}$ by Borthakur et al. (61). Generally, three-dimensional $T_{1 \rho}$ sequences have higher SAR because of the repetitive application of the hard pulses in the SL irradiation cluster, and are limited by the required $B_{1}$ of the SL pulse. A three-dimensional $T_{1 \rho}$ map of a specimen of bovine patella was calculated by Borthakur et al. (61) according to the pixel-by-pixel fitting method. The variation in estimated $T_{1 \rho}$ as a function of $T_{1}$ was calculated, and it was concluded that $\mathrm{a} \pm 10 \%$ error in $T_{1}$ results in less than $\mathrm{a} \pm 3 \%$ change in the calculated $T_{1 \rho}$. Three contiguous 1.5 -mm-thick slices from a three-dimensional dataset of 16 slices from the knee joint of a healthy 30-year-old volunteer in the sagittal plane are shown in a study by Borthakur et al. (61). A clear delineation between cartilage and other tissues, such as bone marrow, was observed inside the proximal tibia and distal femur using the $B_{1}$ value of the SL pulse at $400 \mathrm{~Hz}(61)$.

A sequence that combines $T_{1 \rho}$ and $T_{2}$ quantification in human knee cartilage with a scan time of less than $10 \mathrm{~min}$, and is robust to $B_{0}$ and $B_{1}$ inhomogeneity, was developed with excellent repeatability by Li et al. (62). The sequence was developed based on the magnetization-prepared angle-modulated partitioned $k$-space spoiled gradient echo snapshots (MAPSS) $T_{1 \rho}$ quantification sequence. Composite tip-down pulses were employed to bring the magnetization close to the $\mathrm{SL}$ axis, and the composite tip-up pulses returned the magnetization close to the longitudinal axis even under the condition of a large off-resonance (63). The tip-down/tip-up $90^{\circ}$ hard pulses were followed and preceded by a hard $135^{\circ}$ pulse with the opposite phase. Recently, a three-dimensional gradient echo $T_{1 \rho}$ sequence was used in a study to demonstrate that quantitative $T_{1 \rho}$ mapping may provide a useful biomarker for the assessment of disease progression in Huntington's disease (64), as shown in Fig. 12. Another recent study based on the three-dimensional gradient echo $T_{1 \rho}$ sequence has shown that $T_{1 \rho}$ values are elevated in the cerebral white matter and cerebellum of patients with bipolar disorder (65). A $T_{1 \rho}$-prepared gradient echo sequence, which is capable of obtaining three $T_{1 \rho}$ images with different TSL in a segmented fashion within a single breath-hold, has been used to detect fibrosis in patients with hypertrophic cardiomyopathy (66).

Two-dimensional single-slice (SS) and multi-slice (MS) spin echo sequences

Two-dimensional spin echo sequences usually generate higher signal-to-noise ratio (SNR) than three-dimensional gradient echo 


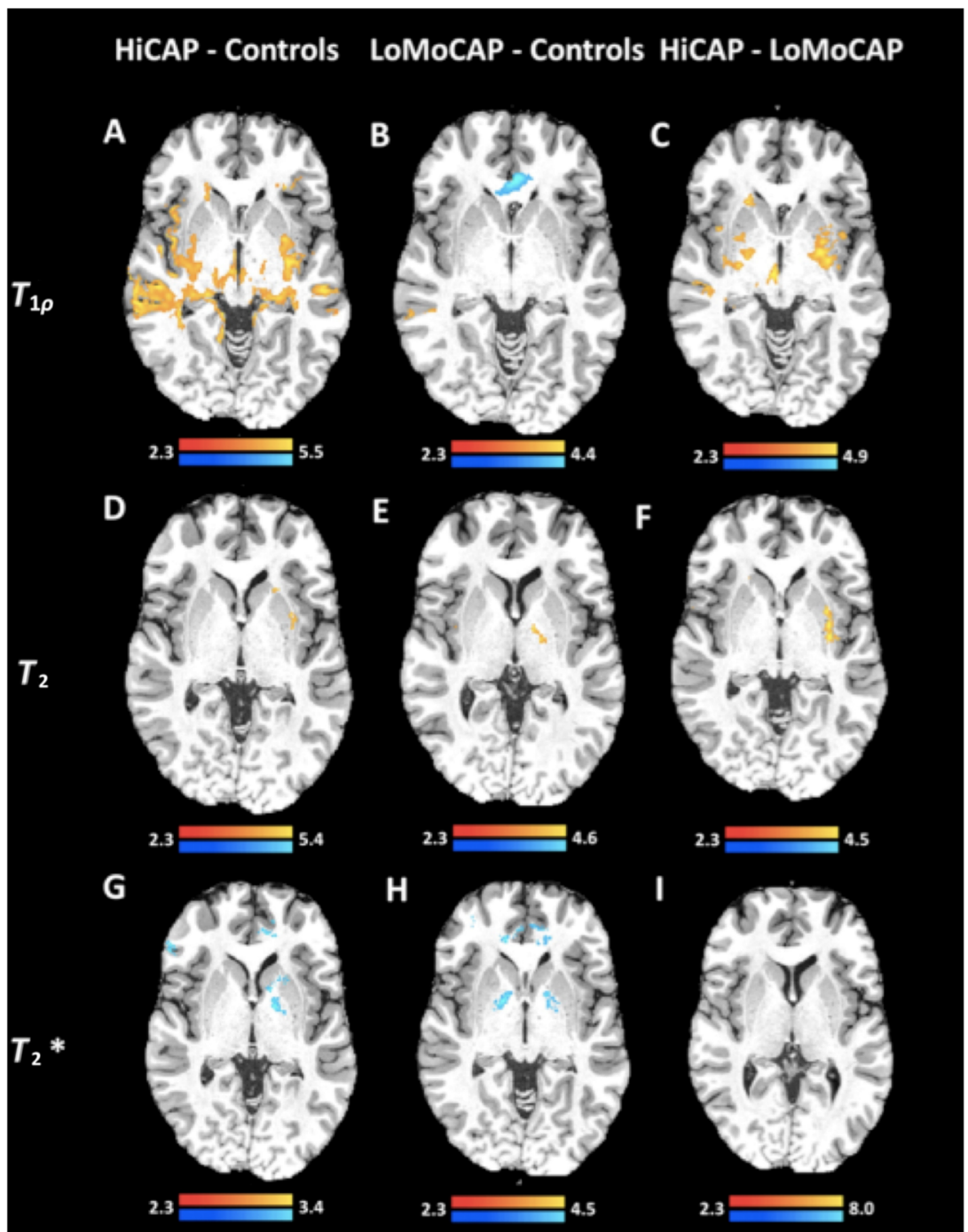

Figure 12. $T_{1 \rho}$ differences between HiCAP subjects [subjects with high CAP value = age $\times$ (cytosine adenine guanine - 33.6), i.e. CAP greater than or equal to 368] and controls (A), LoMoCAP subjects (subjects with low or moderate CAP value $=$ age $\times$ (cytosine adenine guanine -33.6 ), i.e. CAP less than 368 ) and controls (B), and HiCAP and LoMoCAP subjects (C). $T_{2}$ differences between HiCAP subjects and controls (D), LoMoCAP subjects and controls (E), and HiCAP and LoMoCAP subjects (F). $T_{2}{ }^{*}$ differences between HiCAP subjects and controls (G), LoMoCAP subjects and controls (H), and HiCAP and LoMoCAP subjects (I). The $Z$ value maps, thresholded at a $Z$ value of $2.3(p<0.05$, corrected at the cluster level), are overlaid on the Neuroimaging Analysis Center (NAC) atlas for reference. The yellow color indicates prolonged relaxation times, whereas the blue color indicates shorter relaxation times. [Adapted from ref. (64).]

sequences per unit time. The spin echo sequence-based imaging technique for the depiction of human cartilage and muscle abnormalities was designed by Duvvuri et al. (67). It consisted of an SL pulse cluster pre-encoded to the FSE acquisition. The limiting factor of this approach was SS acquisition because of the non-slice-selective RF pulses. Another spin echo sequence capable of acquiring multiple slices was proposed by Wheaton et al. (68), who demonstrated phantom and mouse brain imaging using this sequence. In this method, TSL duration is the limiting factor; however, it can produce good SNR for certain TSL periods $(0-100 \mathrm{~ms})$. For long TSL periods, signal decay caused by persistent saturation can occur, but a self-compensating SL pulse can be used to prevent this decay. Another limitation of this approach was that the images contained both $T_{1 \rho}$ and $T_{2 \rho}$ weighting coupled together as a function of TSL. $T_{2 \rho}$ saturation must be eliminated to perform an accurate and precise quantification of $T_{1 \rho}$ in heterogeneous human tissue. In a study by Wheaton et al. (68), non-selective SL pulses were used and the saturated longitudinal magnetization was modeled and measured independently as $T_{2 \rho}$ decay, and then corrections for $T_{1 \rho}$ measurements were subsequently performed. In this study, normalized average signal intensities, with and without saturation correction, as a function of TSL for the five-slice MS-SL data were compared with SS-SL data (SS-SL-generated $T_{1 \rho}$ values were verified using a spectroscopic method) (68). The average $T_{1 \rho}$ measurement with saturation reduction was nearly the same as the true measurement of $T_{1 \rho}$. The signal intensity of the in vivo image of mouse brain measured using the MS-SL sequence was less than the intensity measured using the SS-SL sequence as a result of saturation from the repeated application of the SL pulses. The SS-SL and MS-SL images had identical $T_{2}$ and $T_{1 \rho}$ weighting, whereas the MS-SL image had an additional decay factor of $T_{2 \rho}$ because of the saturation effect, particularly at long TSL. Possible local $T_{2 \rho}$ variations were accounted for because the $T_{1 \rho}$ measured values were consistent with spectroscopy measurements for the homogeneous sample. Biological tissues 
have spatially inhomogeneous relaxation properties; the saturation effects should be resolved for each pixel value of the $T_{1 \rho}$ map.

Another technique was described by Wheaton et al. (69) for the elimination of the intrinsic $T_{2 \rho}$ weighting from the MS $T_{1 \rho}$ measurements of knee joints of healthy human subjects using a separate single scan. In this method, a single slice was excited by the $T_{1 \rho} \mathrm{SL}$ module, which acted as the saturation pulse to produce the longitudinal magnetization decay as a function of pulse duration according to $T_{2 \rho} . T_{1}$-weighted MS data and $T_{2 \rho}$-weighted SS data were separately obtained in one session. $T_{2 \rho}$ values were estimated by the equations described by Wheaton et al. (69), and the values calculated directly using the data obtained with the sequence were considered as true values. $T_{1 \rho}$ and $T_{2 \rho}$ values were obtained from healthy human patellae (six subjects and 10 slices) using an SL pulse amplitude of $500 \mathrm{~Hz}$ (five evenly spaced TSL values from 10 to $50 \mathrm{~ms}$ ) at 1.5 T. TSL values between 10 and $50 \mathrm{~ms}$ were used for the acquisition of 10 slices. It is important to recognize that this SS method was used to obtain $T_{2 \rho}$ data; therefore, MS $T_{2 \rho}$ saturation elimination of the SL data was not possible using this method. One possible strategy is to acquire the $T_{2}$ data with an MS multi-echo sequence together with the $T_{1 \rho}$ data. Because $T_{2 \rho}$ is more closely related to $T_{2}$, the calculation of $T_{2 \rho}$ using the $T_{2}$ data is generally insensitive for a broad range of $T_{1}$ values. However, if the variations in $T_{1}$ are within a physiologically relevant range, an inaccurate $T_{1}$ will result. This potential source of inaccuracy may be reflected prominently in tissues with short $T_{1}$ or in the presence of severe pathological changes that can affect $T_{1}$.

The quantification of multi-component rotating frame relaxation is affected by variation of the SL field strength in the range $0.5-5 \mathrm{kHz}$. The values of the three resolved $T_{1 \rho}$ components (corresponding to the three-compartment model) increase at high
SL fields and closer orientation to the magic angle $(29,70)$. The smallest number of $T_{1 \rho}$ components and the largest $T_{1 \rho}$ values were obtained in the range in which the SL field is strongest and the dipolar interaction is lowest. At the magic angle, $T_{1 \rho}$ relaxation at the SL field $>1000 \mathrm{~Hz}$ has only one high-value component. This transition between multiple components and a single component manifests when sufficient exchange processes exist that are able to diminish the difference between the different pools of water molecules when the dipolar interaction is minimal at the magic angle.

A method to measure proton exchange in human patella was described by Kogan et al. (71), which combines CEST and $T_{1 \rho}$ magnetization preparation methods (chemical exchange saturation transfer in rotating frame, CESTrho). A slightly modified SL irradiation pulse cluster was employed for the $T_{1 \rho}$ magnetization preparation in this study, in which a refocusing pulse was used between two rectangular SL pulses. The CEST magnetization preparation module consisted of a series of off-resonance saturation pulses, which was followed by the SL irradiation pulse cluster. A two-dimensional turbo spin echo (TSE) readout was used for magnetization acquisition.

Recently, a three-dimensional TSE $T_{1 \rho}$ sequence, with isotropic high-resolution whole-brain coverage, has been applied to assess degenerative changes in multiple sclerosis (72), as shown in Fig. 13. $T_{1 \rho} \mathrm{MRI}$ demonstrates enhanced lesion contrast compared with $T_{2}$ and may provide a useful measure of demyelinating processes in multiple sclerosis. In addition, another study used a three-dimensional TSE $T_{1 \rho}$ sequence to investigate the variations in human brain $T_{1 \rho}$ values over adulthood, and concluded that the $T_{1 \rho}$ relaxation time constant is sensitive to the changes related to the normal aging process (73). A representative $T_{1 \rho}$ map of pediatric articular cartilage in the knee generated using a two-dimensional TSE sequence is shown in Fig. 14.

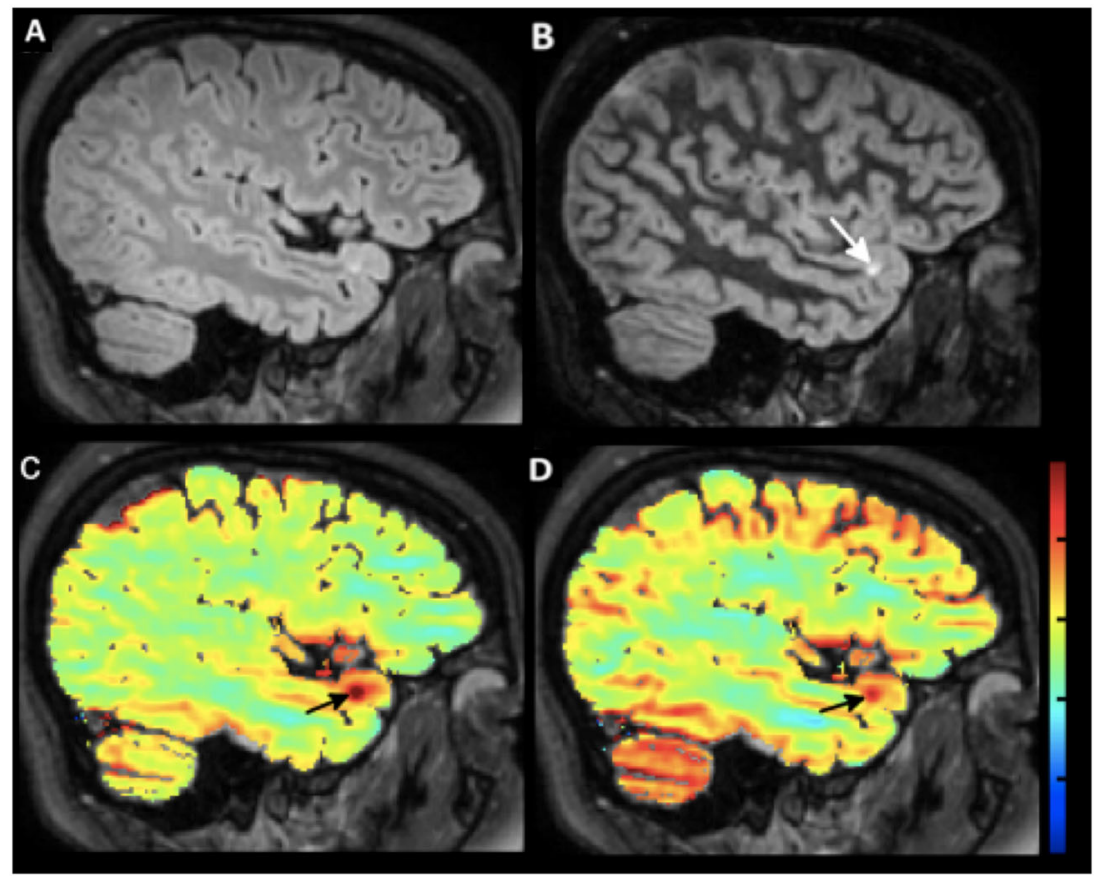

Figure 13. Patient with relapsing-remitting multiple sclerosis with cortical lesion (arrows). (A) Fluid attenuated inversion recovery (FLAIR). (B) Double inversion recovery (DIR). (C) $T_{1 \rho}$ map (range, 50-100 ms). (D) $T_{2}$ map (range, 40-80 ms). The lesion is better visualized on DIR compared with FLAIR and has higher contrast-to-noise ratio (CNR) on the $T_{1 \rho}$ map compared with the $T_{2}$ map. [Adapted from ref. (72).] 


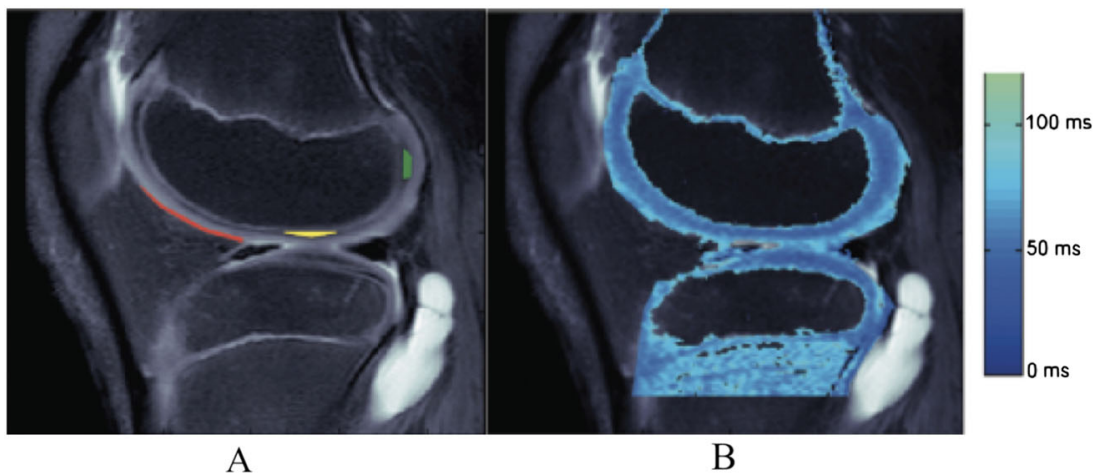

Figure 14. (A) A $T_{2}$-weighted turbo spin echo (TSE) sagittal scan of the knee used to plan the region of interest (ROI) for the $T_{1 \rho}$ map shown in (B). The red $\mathrm{ROI}$ is articular cartilage, the yellow ROI is load-bearing (LB) epiphyseal cartilage and the green ROI is non-load-bearing (NLB) epiphyseal cartilage. (B) $T_{1 \rho}$ map of the same subject as shown in (A). A maximum threshold of $120 \mathrm{~ms}$ was applied to the $T_{1 \rho}$ map, and the soft tissue and patellar regions were removed for clarity. The color scale is given in milliseconds. Note a difference of approximately $25 \mathrm{~ms}$ in articular and epiphyseal cartilage $T_{1 \rho}$ values. [Adapted from ref. (74).]

\section{Echo planar imaging (EPI) sequences}

A sequence termed gradient echo spin-locked EPI (SL-EPI) was introduced by Borthakur et al. (75) for imaging the human brain and blood in the popliteal artery. SL-EPI was used to study the dependence of $T_{1 \rho}$ on in vivo blood oxygenation levels in a clinical setting, and the relative contribution of susceptibility to the relaxation times was different for the $T_{2}, T_{2}{ }^{*}$ and $T_{1 \rho}$ sequences. Comparison of human brain data acquired using SL-EPI, EPI and SL-TSE, with different TE and TSL + TE, obtained at 1.5T, was performed by Borthakur et al. (75). The authors concluded that the $T_{1}$-weighted images were less prone to susceptibility artifacts than $T_{2}{ }^{*}$-weighted images. In this EPI sequence, the effect of the SL pulse on the transverse magnetization was essentially similar to a series of $180^{\circ}$ pulses in a CP train, where the pulses were separated by the inverse of the $B_{1}$ frequency, which was $1 / 500 \mathrm{~Hz}$ or $2 \mathrm{~ms}$ in this study. According to the calculation of SNR as a function of TE for $T_{2}{ }^{*} \mathrm{EPI}$ and TSL + TE for $T_{1 \rho}$ SL-EPI, performed by Borthakur et al. (75), the SL-EPI values were closer to those calculated using SL-TSE. $T_{1 \rho}$ of cerebrospinal fluid (CSF) was slightly lower in SL-EPI than in SL-TSE because of the volume averaging of periventricular tissue with CSF. This sequence was limited to the acquisition of only one slice per $T R$, because the SL pulse was not slice selective. SAR can be reduced by inserting only $S L$ pulses between the excitation pulse and the encoding gradients in the EPI sequence. $\mathrm{A} T_{1 \rho}$ spin echo EPI signal can be generated by placing an $180^{\circ}$ refocusing pulse after the $90^{\circ}$ excitation and SL pulses. Integrating SL pulses with the interleaved EPI sequences (for both gradient echo EPI and spin echo EPI) may discern the relative contributions to the blood oxygenation level-dependent (BOLD) effect from blood flow and blood oxygen saturation changes, which occur in the arterial, capillary and venous pools (75) under specific conditions, such as breath-hold, hypoxia or hypercapnia.

The BOLD signal, measured using a $T_{1 \rho}$ sequence, has both vascular and tissue-associated components. A local volume redistribution effect in an imaging voxel can be the main source of the vascular-associated component because of the large differences between the $T_{1 \rho}$ values of blood, tissue and CSF. In particular, vessel dilation would lengthen the parenchymal apparent $T_{1 \rho}$, but would result in shorter $T_{1 \rho}$ values at the cortex boundary and CSF. The tissue-associated component is probably caused by an activation-induced change in tissue metabolism. Tissue $T_{1 \rho}$ has better localization to the middle cortical layer than the spin echo BOLD response and is faster than both BOLD and cerebral blood volume. Tissue $T_{1 \rho}$ remains unaffected by tissue oxygenation, cerebral blood flow and volume changes induced by hyperoxia (76). The vascular contribution to the $T_{1 \rho}$ functional MRI signal change is $B_{0}$ dependent because the difference in $T_{1 \rho}$ between the blood, tissue and CSF is dependent on $B_{0}$. Similarly, tissue-associated $T_{1 \rho}$ changes, if they were mainly caused by the proton exchange effect, would also be $B_{0}$ dependent, because an increase in $B_{0}$ would result in larger chemical shifts between labile protons and water.

A spin echo EPI acquisition was combined with SL and CEST irradiation pulse cluster by Jin et al. (77) to perform SL and CEST experiments at various $\mathrm{pH}$ levels using concentrated metabolite phantoms with exchangeable amide, amine and hydroxyl protons at 9.4 T. The authors showed that in the intermediate exchange regime, on-resonance SL was the most sensitive to chemical exchanges and detected hydroxyl and amine protons on a millimolar concentration scale. It has also been shown that off-resonance SL and CEST methods are sensitive to slowexchanging protons when an optimal SL or CEST saturation pulse power, respectively, matches the exchange rate. In addition, it has been observed that the offset frequency-dependent SL and CEST spectra are very similar and can be explained using the SL model developed by Trott and Palmer (45). Offset frequency-dependent SL, CEST spectra or on-resonance SL relaxation dispersion measurements have proven to be useful for the determination of the exchange rate and population of metabolite protons. An effective exchange relaxation rate $R_{\mathrm{ex}}$ was constructed from the CEST $z$ spectra to quantitatively characterize the chemical exchange process. A simple and conventional measure of chemical exchange contrast, MTR asym, was employed and the CEST saturation pulse power determined the degree of asymmetry of MTR asym. Because MTR asym is not a monotonic function of exchange rate and $\mathrm{pH}$, its application in the intermediate exchange regime becomes problematic.

\section{Balanced steady-state sequences}

There are a few $T_{1 \rho}$ steady-state sequences that employ short delay times with the $T_{1 \rho}$-prepared three-dimensional acquisition schemes. These sequences are inherently time inefficient because they employ delay periods to wait for the equilibrium 
restoration of the longitudinal magnetization. They allow partial recovery of the longitudinal magnetization, as mentioned previously in the study by Li et al. (62), and full recovery in the study by Witschey et al. (78). The inability to attain high SNR, the long acquisition time and the high SAR are the major limitations of these approaches. Therefore, it is important to acquire the $T_{1 \rho}$ weighted magnetization under rapid and balanced steady-state conditions to achieve high SNR and contrast with lower SAR. The balanced gradient echo steady-state (b-GRE) sequence used by Witschey et al. (78) was capable of combining standard $T_{1 \rho} \mathrm{SL}$ pulse cluster with balanced steady-state free precession (b-SSFP), FIESTA, balanced fast field echo (b-FFE) or true fast imaging with steady-state precession (True-FISP). This b-GRE acquisition was used to obtain three-dimensional coverage of the patellofemoral and tibiofemoral cartilage and lower lumbar spine of a human asymptomatic subject. The $T_{1 \rho}$ preparation period and balanced steady-state acquisition period are inherently conflicting concepts, because the former enables $T_{1 \rho}$ weighting of the equilibrium magnetization, whilst the latter abolishes any previous weighting during the approach from the transient state to the steady state via transient signal decay during acquisition. This sequence could acquire a single view (axial, coronal or sagittal) in one-third of the time necessary to acquire similar data using the $T_{1 \rho}$-prepared three-dimensional gradient echo sequence. This sequence begins with the application of a pulse cluster, which includes a CW-SL pulse amplitude in the range 200$1000 \mathrm{~Hz}$, and the residual magnetization in the transverse plane is destroyed after the SL pulse cluster. For other applications, this pulse cluster can be preceded by a slab-selective inversion recovery pulse or a spectrally selective fat saturation pulse and spoiler gradient. To prepare the balanced magnetization state, another pulse cluster was applied, which was composed of a small flip angle RF pulse, delay time period and dummy RF pulses. Thereafter, b-GRE image encoding for a specific phaseencoding line was performed, followed by a longitudinal relaxation delay time period to restore equilibrium magnetization. These steps were repeated for the next phase-encoding line. This method was based on the acquisition of the SL magnetization during the transient period of the b-GRE signal evolution, where the memory of the SL magnetization was retained. A blurring problem was associated with this type of acquisition because the transient signal decay filtered the $k$ space in the phaseencoding direction (17). Variable flip angle and $T_{2}$-TIDE (79) based schemes have been proposed to solve this problem; however, the variable flip angle method should be customized for a particular tissue and the $T_{2}$-TIDE method blurs the signal as a result of $T_{2}$ decay during the initial phase of acquisition. Flip angle optimization and $k$-space filtering methods were used by Witschey et al. (78) to reduce image blurring. Subsequently, $T_{1 \rho}$ mapping of human patellar and femoral cartilage and the human spine in vivo was demonstrated by Witschey et al. (78).

Another SL steady-state free precession (sISSFP) pulse sequence was proposed by Witschey et al. (80) to measure $T_{1 \rho}$, and compared the sISSFP signal with the well-known b-SSFP signal originating from an $\mathrm{MnCl}_{2}$-doped phantom and the human brain at 7T. This technique was based on three-dimensional non-selective excitation using typical off-resonance hard SL pulses applied from TR to TR, interrupted briefly for a short period of a typical b-SSFP acquisition regime. sISSFP imaging can be performed with various RF pulse power values, without significant changes in contrast, under extreme motional narrowing conditions.
A rotary echo SL pulse cluster was integrated with a twodimensional b-FFE sequence at $3 \mathrm{~T}$ by Yuan et al. (81). To force a fast approach to steady state, an $\alpha / 2$ pulse was applied before the subsequent $\alpha$ pulses in a typical b-SSFP sequence, where $\alpha$ is the flip angle for data acquisition. The $\alpha / 2$ pulse and four startup pulses were used by Yuan et al. (81) during the course to steady state, whilst maintaining the $T_{1 \rho}$ preparation. A normal phase alternating b-FFE readout and centric acquisition order were employed to maintain the $T_{1 \rho}$ weighting of images during the transient state. Eighty $k$-space lines were acquired for each shot. A delay time of $5000 \mathrm{~ms}$ after each shot was set to restore equilibrium magnetization prior to the next $T_{1 \rho}$ preparation. It is important to note that only six slices were acquired using this sequence. The precision in $T_{1 \rho}$ estimation with two SL times was analyzed by Yuan et al. (81), and the feasibility of using two specific operator-selected TSLs for precise and accurate liver $T_{1 \rho}$ mapping was examined. No significant difference was observed between the $T_{1 \rho}$ maps generated using the optimized two-TSL (1 and $50 \mathrm{~ms}$ ) combination and the maps generated using the six TSLs of $1,10,20,30,40$ and $50 \mathrm{~ms}$ at an SL frequency of $500 \mathrm{~Hz}$. The $B_{0}$ and $B_{1}$ field inhomogeneity artifacts may compromise the robustness and reliability of using a limited number of TSLs, specifically at low SL frequencies. An analytical expression of $T_{1 \rho}$ magnetization for rotary echo SL pulses was derived by Yuan et al. (82) to demonstrate its relationship with the off-resonance frequency, TSL, SL frequency, and $T_{1 \rho}$ and $T_{2 \rho}$ relaxation time constants. A high SNR is critically important for high-precision $T_{1 \rho}$ mapping with limited TSLs. The average SAR was not reduced by reducing TSLs because, in terms of RF energy deposition, the SL RF SAR level is proportional to the square of the flip angle of SL pulses within the unit pulse duration, equivalent to the SL frequency. Nevertheless, the total RF energy deposited by the SL pulses was reduced by a factor of approximately 3 in that sequence. However, the utilization of sISSFP with different TSL durations remains unexplored. For a fixed RF amplitude, $\omega_{1}$, the tradeoff between $T_{1}$ and $T_{1 \rho}$ contrast, which depends on $\Delta \omega$, remains to be explored. If hard RF pulses are used for SL excitation, the transient decay to the steady state is affected by the $B_{0}$ and $B_{1}$ inhomogeneity, and the magnetization can attain a different steady state as a result of the free precession that occurs from $B_{0}$ inhomogeneity. There is no appropriate description regarding the energy exchange effects around the lattice in the rotating frame of reference under the b-SSFP regime. A thorough study is required to analyze the effects of different variables of an RF irradiation scheme, i.e. the exchange rates, number and size of spin pools undergoing exchange and $\omega_{1}$-based disentangling of the $T_{1 \rho}$ dispersion effect from the MT effect. The quantitative relationship between the frequency-dependent response of the SISSFP sequence, the effective field orientation, SL pulse amplitude, $\Delta \omega$ and the relaxation rates has not yet been established.

\section{Sequences based on adiabatic pulses}

The relative changes in the measured relaxation rate constants generated by varying the parameters of CW-SL and adiabatic SL irradiation were calculated by Mangia et al. (41). For adiabatic excitation, HS1 or HS4 adiabatic pulses were placed at the start of the FSE readout imaging sequence. For CW-SL excitation, a CW pulse was inserted between two AHP pulses prior to FSE. In order to compare optimal parameters (flip angle, pulse duration, modulation functions, bandwidth, rotational and exchange 
correlation times) for the measurement of $R_{1 \rho \text {-dd }}$ and $R_{1 \rho \text {-ex }}$ in the case of CW and adiabatic irradiation, simulations were used and the results were in agreement with the human brain in vivo measurements at $4 \mathrm{~T}$ (41). The relaxations during the CW-SL excitation were calculated according to the expressions derived in several studies $(39,40,43,44)$. The relaxations during the adiabatic SL rotation in the weak field approximation were calculated as an average of the instantaneous time-dependent contributions from the different relaxation mechanisms $(42,46,47)$. The abovementioned methods developed by Mangia et al. (41) were used to reveal abnormalities in normal-appearing white matter of subjects with multiple sclerosis (83).

The implementation of the CW-SL sequence for in vivo clinical applications is challenging for several reasons, such as increased $B_{0}$ inhomogeneities with increased $B_{0}$ strength and the tilt angle uncertainty caused by variations in $B_{1}$. At high magnetic field, high locking power is required for sufficient spin locking. There are SAR limitations on the range of $\omega_{1}^{\max }$. The use of the adiabatic $\mathrm{SL}$ sequence is dependent on suitable values of the bandwidth of the pulse functions and $\omega_{1}^{\max }$ with respect to in vivo conditions. The minimum values achievable for adiabaticity can be beyond the required range of values for human imaging. Therefore, the contrast in adiabatic SL sequences is usually manipulated using different pulse modulation functions. $T_{1 \rho}$ imaging has been performed within SAR guidelines in the human brain using adiabatic pulses in several studies $(42,54)$. The corresponding SL excitation schemes are illustrated in Fig. 11. The method developed by Michaeli et al. (42) was used to demonstrate that the sub-millimeter resolution $T_{1 \rho}$ and $T_{2 \rho}$ relaxation time constants were a non-invasive measure of iron content, as well as evidence of neural loss in the mid-brain of patients with Parkinson's disease (9), as shown in Fig. 15.

Ultrashort TE sequences

An ultrashort TE (UTE) $T_{1 \rho}$ sequence was employed by Du et al. (84) to measure $T_{1 \rho}$ of goat posterior cruciate ligament and hu- man Achilles tendon specimens at a series of angles relative to the $B_{0}$ field and SL field strengths to investigate the contribution of dipole-dipole interactions to $T_{1 \rho}$ relaxation. Significant magic angle effects were observed by the increase in $T_{1 \rho}$ in the posterior cruciate ligament near the magic angle. In a study by Du et al. (85), another UTE sequence based on the dual inversion recovery method was used to quantify the zone of calcified cartilage.

\section{Variations in SL field and $T_{1 \rho}$ dispersion imaging studies}

$T_{1 \rho}$ dispersion in the human brain in vivo was demonstrated by Borthakur et al. (86) using an FSE-based sequence. This method was employed by Haris et al. to evaluate the $T_{1 \rho}$ relaxation time constant in hippocampus in the brain of patients with Alzheimer's disease (87), as shown in Fig. 16, and in patients with Parkinson's disease (88). It was concluded by Virta et al. (28) that $R_{1 \rho}$ was sensitive to the molecular weight, concentration and structure of the protein, using on-resonance SL MRI for SL fields below $200 \mu \mathrm{T}$ and at $B_{0}=0.1 \mathrm{~T}$. In addition, it was observed that the amount of intra- and intermolecular hydrogen and disulfide bonds particularly contributed to $R_{1 \rho}$. $T_{1 \rho}$ dispersion imaging was performed on organs with high macromolecular protein content, such as muscle (anterior tibia and gastrocnemius), at an SL range of $10-160 \mu \mathrm{T}$ and $B_{0}=0.1 \mathrm{~T}$ (14). The comparison of normal and diseased muscle by Virta et al. (15) revealed that $R_{1 \rho}$ of fat was $B_{1}$ field independent, whereas $R_{1 \rho}$ of muscle clearly decreased with an increasing $B_{1}$ field. In the SL range of $50-460 \mu \mathrm{T}$ at $0.02 \mathrm{~T}$, fresh brain infarctions had a long $T_{1 \rho}$ and smaller $T_{1 \rho}$ dispersion than normal brain tissue because of the higher water content and reduced macromolecular fraction in a fresh infarction (25). $T_{1 \rho}$ dispersion was employed to differentiate between benign and malignant head and neck tumors $(34,89)$. Considerable $T_{1 \rho}$ dispersions were observed in brain tissues in the SL range of $50-250 \mu \mathrm{T}$ (2) and in head and neck tissues in the SL range of 35-100 $\mu \mathrm{T}$ (89). However, the $T_{1 \rho}$ dispersions in the SL

\section{PD Patient}
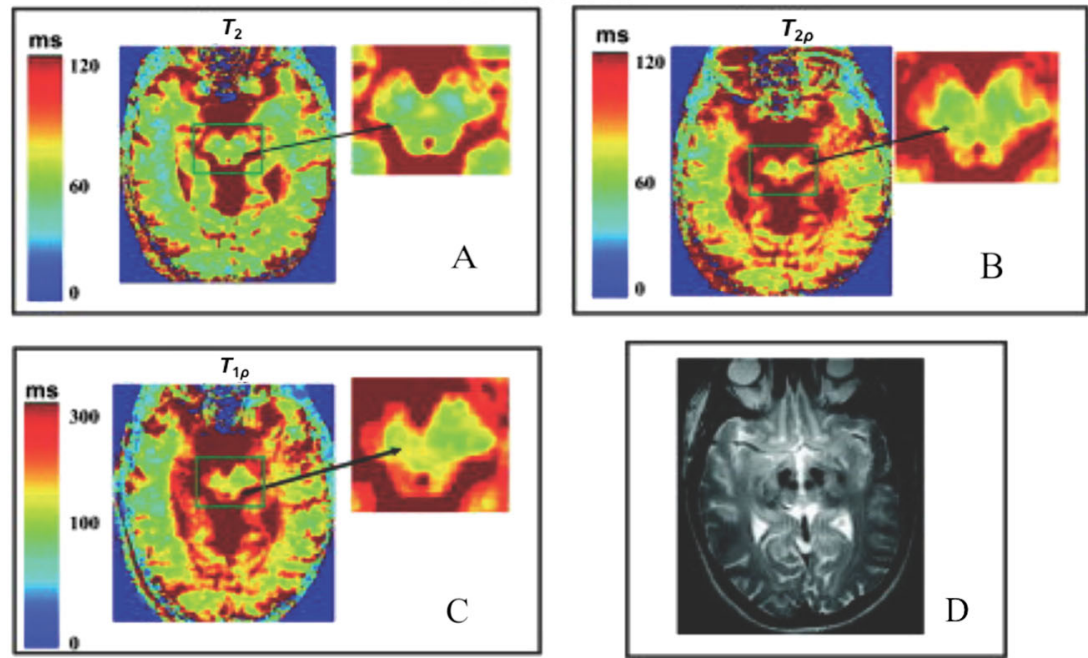

Figure 15. $T_{2}(\mathrm{~A}), T_{2 \rho}$ (B) and $T_{1 \rho}$ (C) maps generated from a transverse slice in a patient with Parkinson's disease. (D) $T_{2}$-weighted image of the same slice acquired with a rapid relaxation enhancement pulse sequence. $T_{2 \rho}$ images were obtained with no time delays between adiabatic full passage (AFP) in the AFP pulse train placed after the adiabatic half passage pulse. $T_{1 \rho}$ images were obtained with no time delays between AFP in the AFP pulse train placed prior to the imaging readout. $T_{2}$ images were measured with the double spin echo pulse sequence using a variable interpulse time interval. For $T_{1 \rho}, T_{2 \rho}$ and $T_{2}$ measurements, the TurboFLASH imaging readout (four segments) was used. Images were measured using an in-plane resolution of $0.70 \mathrm{~mm}^{2}$, field of view of $20 \mathrm{~cm}^{2}, 2562$ matrix, slice thickness of $3 \mathrm{~mm}$ and TR=4.5 s. [Adapted from ref. (9).] 

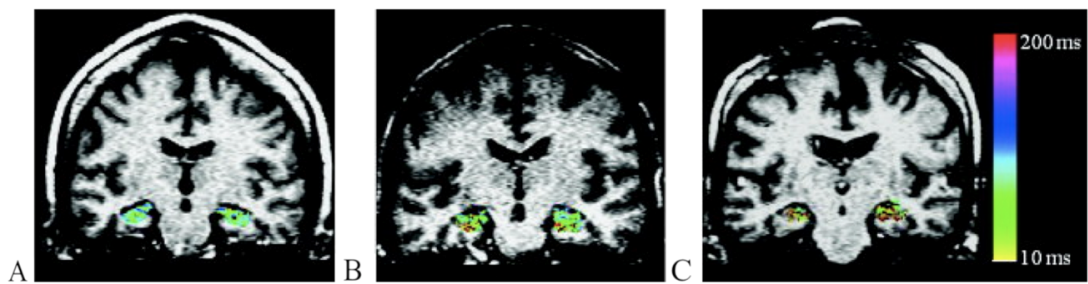

Figure 16. $T_{1 \rho}$ maps of the hippocampus region in the brain (in color) overlaid on fluid-attenuated $T_{1 \rho}$ MRI of control [female, 72 years (A)], mild cognitive impairment [male, 76 years (B)] and Alzheimer's disease [female, 75 years (C)]. Pixels with higher $T_{1 \rho}$ (red) are more prominent in the hippocampus of the patient with Alzheimer's disease. A lack of signal from cerebrospinal fluid (CSF) implies that the higher $T_{1 \rho}$ values are not caused by fluid. [Adapted from ref. (87).]

range of $50-250 \mu \mathrm{T}$ of different healthy brain regions were similar and thus not useful for healthy brain matter discrimination.

The $T_{1 \rho}$ dispersion profile of rat tissues in vitro measured at $0.1 \mathrm{~T}$ by Koskinen et al. (90) had a similar pattern in various tissues (liver, brain, spleen, kidney, heart and skeletal muscle). The highest $R_{1 \rho}$ values were measured in the liver and muscle, followed by the heart, whereas the values for spleen, kidney and brain were similar. The $T_{1 \rho}$ dispersion of subcutaneous fat was measured by Virta et al. (14) and no conspicuous dispersion was observed. Because the SL pulse amplitude is dependent on the Larmor frequency $\left(\gamma B_{1} / 2 \pi\right), R_{1 \rho} \sim R_{2}\left(1 / T_{2}\right)$ at values of $\gamma B_{1} / 2 \pi$ near zero and $R_{1}$ increases to a maximum of $R_{1}\left(1 / T_{1}\right)$ as the value of $\left(\gamma B_{1} / 2 \pi\right)$ increases (52). The corresponding dispersion reflects the characteristic time-scale of the irreversible dephasing effects.

\section{$T_{2 \rho}$ sequences and their applications}

Two-dimensional SS spin echo sequences

A $T_{2 \rho}$-weighted two-dimensional $\mathrm{SS}$ spin echo sequence was described by Wheaton et al. (52). Mean $T_{2}$ and $T_{2 \rho}$ values of the human brain structures (white matter, gray matter, putamen and caudate nucleus), single subject and SS acquisition, were calculated using an SL pulse amplitude of $500 \mathrm{~Hz}$ (five evenly spaced TSL values from 15 to $75 \mathrm{~ms}$ ) at $1.5 \mathrm{~T}$. The measured $T_{2}$ values appeared to be $50 \%$ greater than the $T_{2}$ values for all regions of the brain. $T_{2 \rho}$ contains the auxiliary influence of $T_{1}$, which can potentially aid in the differentiation of tissue structures with similar $T_{2}$. This difference in $T_{2 \rho}$ translates into a $38 \%$ increase in contrast between the tissues in the $T_{2 \rho}$-weighted image compared with the $T_{2}$-weighted image. The increased contrast between general types of tissue can be used to improve the accuracy and precision of volumetric quantification in the brain.

\section{Sequences based on adiabatic pulses}

In a study by Michaeli et al. (46), the quantification of $T_{2 \rho}$ relaxation as a result of chemical exchange during the AFP pulses of a $\mathrm{CP}$ spin echo sequence was performed. Signal intensity decay during the adiabatic $C P$ sequence can be described by exponential decay functions with rate $R_{2 \rho}(t)$ during the pulses and $R_{2}$ during the interpulse time intervals (46). Sensitivity to the chemical exchange and the rotational correlation times was manifested by the HS1 and HS4 adiabatic pulses using different modulation functions for the adiabatic pulses. The theoretical developments and experimental verifications of these techniques have been described in detail in several reports $(42,46,60)$. This method was also applied by Nestrasil et al. (91) to measure $T_{2 \rho}$ values in patients with Parkinson's disease. SAR has been the major obstacle in the development of these techniques for clinical applications. In order to compare optimal parameters (flip angle, pulse duration, modulation functions, bandwidth, rotational and exchange correlation times) for the measurement of $R_{2 \rho \text {-dd }}$ and $R_{2 \rho \text {-ex }}$ in the case of CW and adiabatic irradiation, simulations were used and the results were in agreement with the human brain in vivo measurements at $4 \mathrm{~T}$ (41). In the $T_{2 \rho}$ LASER sequence (92), which was employed for MRS of rat brain at $9.4 \mathrm{~T}$, a CP train of AFP pulses was applied in the presence of gradients for three-dimensional localization, and was phase cycled according to the Malcolm Levitt's composite pulse decoupling (MLEV) scheme. The interpulse delay was $1.5 \mathrm{~ms}$ in $T_{2 \rho}$ LASER (equivalent to the duration of one AFP pulse). In MRS, the data are acquired separately for resonances that are spread over a wide chemical shift range. Therefore, the utilization of CW-SL for MRS applications in vivo is time inefficient because the rotating frame relaxation rates measured during a CW-SL pulse are dependent on the frequency offset between the carrier frequency of the RF pulse and the frequency of the isochromat of interest. In a study by Mangia et al. (93), phantom and in vivo measurements were performed to measure the relaxations during the train of HS AFP pulses as a function of the offset from the carrier frequency. In particular, the adiabatic $R_{1 \rho}$ and $R_{2 \rho}$ values of total creatine and $N$-acetylaspartate were measured using localized MRS in a healthy human brain at 4T. However, adiabatic $R_{1 \rho}$ and $R_{2 \rho}$ values of the methyl protons of total creatine (at $3.03 \mathrm{ppm}$ ) were almost doubled when compared with those of the methyl protons of $\mathrm{N}$-acetylaspartate (at $2.01 \mathrm{ppm})$, despite the fact that these resonances were in the flat region of the inversion band of the AFP pulses. It was concluded that $R_{1 \rho}$ and $R_{2 \rho}$ values were minimally dependent on the frequency offset under general experimental conditions, unlike CW SL measurements. Hence, the dissimilarities in adiabatic $R_{1 \rho}$ and $R_{2 \rho}$ values of human brain metabolites are not caused by their chemical shifts, but instead exemplify differences in dynamics.

The sensitivity to $B_{0}$ and $B_{1}$ inhomogeneity is reduced in the high-order rotating frame RAFF method that was mentioned earlier, but this method is non-slice selective. Two-dimensional MS and three-dimensional sequences using the gradient offsetindependent adiabaticity (GOIA) design (55) were introduced by Andronesi et al. (94). The gradient offset-independent adiabaticity wideband, uniform rate, smooth truncation (WURST) modulation, i.e. GOIA-W $(4,16)$, pulse (95) reduces the maximum $B_{1}$ amplitude by $55 \%$, resulting in $80 \%$ less SAR, compared with conventional adiabatic pulses of the same bandwidth and duration. Alternatively, the GOIA-W $(4,16)$ pulse can still attain lower $B_{1}$ amplitude than conventional adiabatic pulses in the shorter 
duration, which is a useful feature for the $T_{1 \rho}$ and $T_{2 \rho}$ irradiation module. In comparison with a CW-SL of the same amplitude, the GOIA-W $(4,16)$ pulse achieves a $20 \%$ reduction in SAR. Slice selection for $T_{1 \rho}$ and $T_{2 \rho}$ is performed using the gradient modulation in GOIA-W $(4,16)$ pulses. Two-dimensional spin echo EPI and three-dimensional turbo FLASH readouts were used with the GOIA-W $(4,16)$ pulse-based SL irradiation module for robust and feasible $T_{1 \rho}$ and $T_{2 \rho}$ imaging of the human brain (94). GOIA$\mathrm{W}(4,16)$ pulses are concatenated without gaps according to the Malcolm Levitt's composite pulse decoupling-4 (MLEV-4) phase cycling approach (96). For $T_{2 \rho}$ mapping, the GOIA-W $(4,16)$ pulse train is preceded and followed by $90^{\circ}$ pulses. For twodimensional slice-selective $T_{2 \rho}$ mapping, $90^{\circ} \mathrm{SLR}$ pulses are used, whereas, for three-dimensional mapping, $90^{\circ}$ AHP HS8 pulses are used. The parameters of molecular dynamics can be calculated from rotating frame relaxation curves as shown previously in several studies $(42,79,97,98)$. In the study by Andronesi et al. (94), three-dimensional maps of water molecular dynamics in the human brain region (white matter, gray matter, caudate, putamen, thalamus, pallidum, hippocampus and ventral diencephalon) were shown.

\section{SUMMARY OF $T_{1 \rho}$ AND $T_{2 \rho}$ MRI TECHNIQUES}

Since its inception, $T_{1}$-weighted imaging has been used to evaluate the pathology and physiology of brain, breast, liver, muscle and the human musculoskeletal system, whereas $T_{2 \rho}$-weighted imaging has been mainly used for brain imaging. In this review, basic contrast mechanisms and signal generation in $T_{1 \rho}$ and $T_{2 \rho}$ MRI, and the MRI sequences employed for the abovementioned applications, have been discussed. As the imaging sequences are the basic tools for the measurement of rotating frame relaxation rates, the description of technical strengths and limitations is an important feature of this review. Ideally, the application of $T_{1 \rho}$ and $T_{2 \rho}$ mapping sequences to the human body in the clinical environment requires full volume coverage of the desired region of the body. Except for the sequence introduced by Andronesi et al. (94) for human brain imaging, the two-dimensional MS sequences presented to date are insufficient for a standard clinical examination, with incomplete volume coverage as a result of the different factors described above. However, the typical threedimensional sequences require long scan times. Solution of the problems related to the method proposed by Andronesi et al. (94), such as the accumulation of imperfect inversion of the magnetization over the pulses in the presence of the gradient, sensitivity to diffusion in two-dimensional $T_{2 \rho}$ imaging, effects of cross terms from other imaging gradients and the inclusion of autorelaxations, cross-relaxations and cross-correlations in the relaxation model, would increase the accuracy and precision of the whole-brain rotating frame MRI method. A three-dimensional spoiled gradient echo sequence provides full volume coverage using typical SL irradiation, but magnetization saturation reduces the SNR because of the short TR, and it requires compensation of $T_{1}$ weighting. Sequences that employ multiple hyperbolic secant adiabatic pulses either do not provide full volume coverage or SAR exceeds limitations. Application of a slice selection gradient with the off-resonance SL pulse is another challenge because this process would introduce the dephasing effect, which destroys $\mathrm{SL}$ in the direction parallel to the effective field. The ability of the sISSFP sequence to maintain similar contrast levels across different preparatory flip angles and modulations of the effective field orientation is advantageous at high (3T) and ultrahigh $(>7 \mathrm{~T})$ fields, where changes in RF amplitude are required to overcome the SAR constraints. Although new methods are required to provide solutions to the challenging technical problems in order to improve the abovementioned imaging sequences for rotating frame $\mathrm{MRI}, T_{1 \rho}$ imaging has extensive potential to become a accurate and precise measure for early disease diagnosis and progression. The number of clinical applications based on contrast in the rotating frame has increased in the last decade as a result of the numerous improved SL
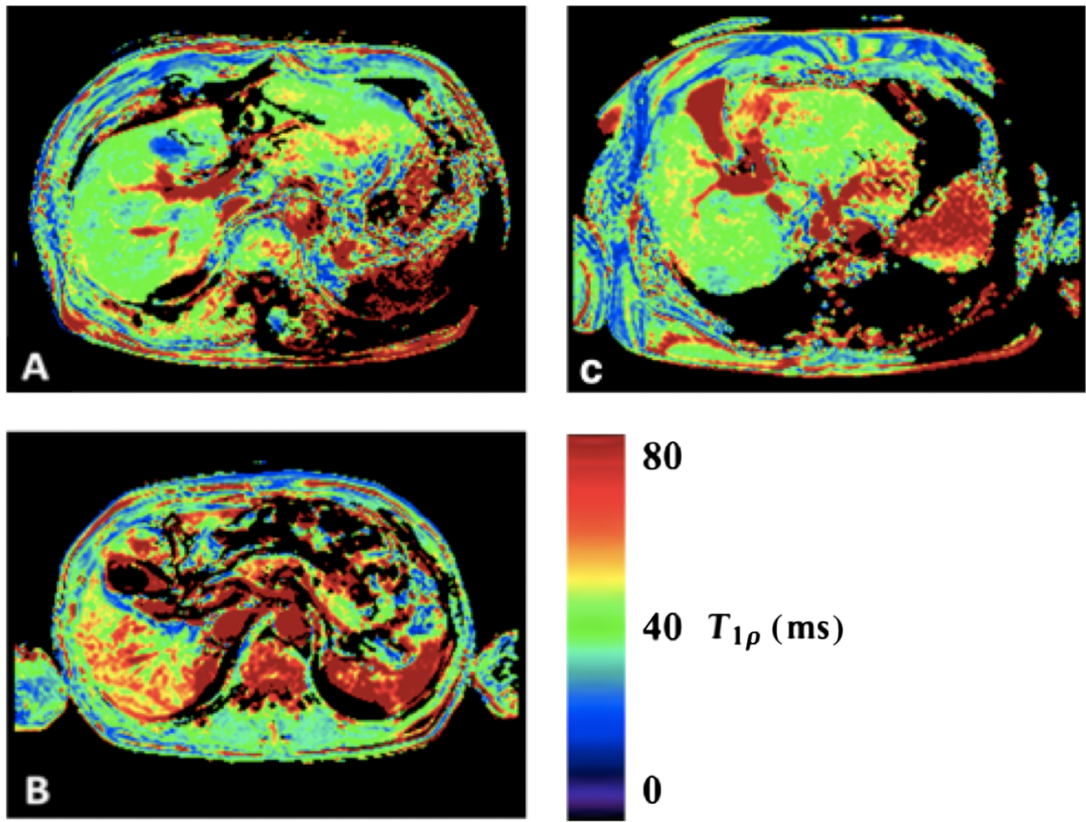

Figure 17. Colored $T_{1 \rho}$ maps. (A) A 68-year-old man, mean $T_{1 \rho}=38.5 \mathrm{~ms}$, indocyanine green (ICG-R15) $=9.3 \%$. (B) A 76-year-old woman, mean $T_{1 \rho}=44.7 \mathrm{~ms}$, ICG-R15 $=40.7 \%$. (C) A 64-year-old man, mean $T_{1 \rho}=54.1 \mathrm{~ms}$, ICG-R15 $=79.9 \%$. The $T_{1 \rho}$ value of the liver was prolonged with increasing ICG-R15, i.e. worsening liver function. [Adapted from ref. (102).] 
irradiation techniques mentioned in this review. A recently published review article on $T_{1 \rho} \mathrm{MRI}$ of the human musculoskeletal system summarizes the practical applications of $T_{1 \rho} \mathrm{MRI}$ methods in this region (99). The recent $T_{1 \rho}$ liver imaging studies show that these techniques can offer clinically significant information associated with biochemical changes in the human liver (100-102), as shown in Fig. 17. The $T_{1 \rho}$ MRI technique may be very useful for myocardial tissue characterization (103). Additional recent applications of these techniques can be found in refs. (74,104116). The main strength of $T_{1 \rho}$ and $T_{2 \rho} \mathrm{MRI}$, i.e. the sensitivity to slow spin motions in tissues, is evident in the literature reviewed in this paper. Therefore, the prospects of clinical applications utilizing the strengths of rotating frame relaxometry in MRI are promising.

\section{Acknowledgements}

Funding was provided by the Academy of Finland to the Brain Research Unit, O. V. Lounasmaa Laboratory for Center of Excellence on Systems Neuroscience and Neuroimaging, Aalto University, Aalto, Finland. Funding was also provided by the National Magnetic Resonance Research Center (UMRAM), Ankara, Turkey.

\section{REFERENCES}

1 Sepponen RE, Pohjonen JA, Sipponen JT, Tanttu Jl. A method for $T_{1 \rho}$ imaging. J. Comput. Assist. Tomogr. 1985; 9(6): 1007-1011.

2 Ramadan UA, Markkola AT, Halavaara JT, Tanttu J, Häkkinen AM, Aronen HJ. On- and off-resonance spin-lock MR imaging of normal human brain at $0.1 \mathrm{~T}$ : possibilities to modify image contrast. Magn. Reson. Imaging 1998; 16(10): 1191-1199.

3 Aronen HJ, Ramadan UA, Peltonen TK, Markkola AT, Tanttu Jl, Jääskeläinen J, Häkkinen AM, Sepponen RE. 3D spin-lock imaging of human gliomas. Magn. Reson. Imaging 1999; 17(7): 1001-1010.

4 Santyr GE, Henkelman RM, Bronskill MJ. Spin locking for magnetic resonance imaging with application to human breast. Magn. Reson. Med. 1989; 12(1): 25-37.

5 Kettunen Ml, Gröhn OHJ, Silvennoinen MJ, Penttonen M, Kauppinen RA. Effects of intracellular $\mathrm{pH}$, blood, and tissue oxygen tension on $T_{1}$ relaxation in rat brain. Magn. Reson. Med. 2002; 48(3): 470-477.

6 Kettunen MI, Gröhn OHJ, Penttonen M, Kauppinen RA. Cerebral $T_{1 \rho}$ relaxation time increases immediately upon global ischemia in the rat independently of blood glucose and anoxic depolarization. Magn. Reson. Med. 2001; 46(3): 565-572.

7 Duvvuri U, Goldberg AD, Kranz JK, Hoang L, Reddy R, Wehrli FW, Wand AJ, Englander SW, Leigh JS. Water magnetic relaxation dispersion in biological systems: the contribution of proton exchange and implications for the noninvasive detection of cartilage degradation. Proc. Natl. Acad. Sci. U. S. A. 2001; 98(22): 12 479-12 484.

8 Borthakur A, Sochor M, Davatzikos C, Trojanowski JQ, Clark CM. T1rho MRI of Alzheimer's disease. Neuroimage 2008; 41(4): 1199-1205.

9 Michaeli S, Oz G, Sorce DJ, Garwood M, Ugurbil K, Majestic S, Tuite P. Assessment of brain iron and neuronal integrity in patients with Parkinson's disease using novel MRI contrasts. Mov. Disord. 2007; 22(3): 334-340.

10 Wang YX, Yuan J, Chu ES, Go MY, Huang H, Ahuja AT, Sung JJ, Yu J. T1rho MR imaging is sensitive to evaluate liver fibrosis: an experimental study in a rat biliary duct ligation model. Radiology 2011; 259(3): 712-719.

11 Stehling C, Luke A, Stahl R, Baum T, Joseph G, Pan J, Link TM. Meniscal $T_{1 \text { rho }}$ and $T_{2}$ measured with 3.0 T MRI increases directly after running a marathon. Skel. Radiol. 2011; 40: 725-735.

12 Hakumäki JM, Gröhn OHJ, Tyynelä K, Valonen P, Ylä-Herttuala S, Kauppinen RA. Early gene therapy-induced apoptotic response in BT4C gliomas by magnetic resonance relaxation contrast $T_{1}$ in the rotating frame. Cancer Gene Ther. 2002; 9(4): 338-345.
13 Azhari H, McKenzie CA, Edelman RR. MR angiography using spinlock flow tagging. Magn. Reson. Med. 2001; 46(5): 1041-1044.

14 Virta A, Komu M, Lundbom N, Kormano M. $T_{1 \text { rho }}$ MR imaging characteristics of human anterior tibial and gastrocnemius muscles. Acad. Radiol. 1998; 5(2): 104-110.

15 Virta $A$, Komu $M$, Lundbom $N$, Jääskeläinen $S$, Kalimo $H$, Airio $A$, Alanen A, Kormano M. Low field T1rho imaging of myositis. Magn. Reson. Imaging 1998; 16(4): 385-391.

16 Halavaara JT, Lamminen AE, Bondestam S, Sepponen RE, Tanttu JI. Spin lock magnetic resonance imaging in the differentiation of hepatic haemangiomas and metastases. Br. J. Radiol. 1995; 68(815): 1198-1203.

17 Halavaara JT, Sepponen RE, Lamminen AE, Vehmas T, Bondestam S. Spin lock and magnetization transfer MR imaging of local liver lesions. Magn. Reson. Imaging 1998; 16(4): 359-364.

18 Cobb JG, Xie J, Gore JC. Contributions of chemical exchange to $T_{1 \mathrm{p}}$ dispersion in a tissue model. Magn. Reson. Med. 2011; 66(6): 1563-1571.

19 Cobb JG, Xie J, Gore JC. Contributions of chemical and diffusive exchange to $T_{1 \rho}$ dispersion. Magn. Reson. Med. 2013; 69(5): 1357-1366.

20 Bloembergen N, Purcell EM, Pound RV. Relaxation effects in nuclear magnetic resonance absorption. Phys. Rev. 1948; 73: 679-712.

21 Fullerton GD. Physiologic basis of magnetic relaxation. In Magnetic Resonance Imaging, Stark DD, Bradley WG (eds). Toronto: Mosby, 1988, pp. 36-55.

22 Daskiewicz OK, Hennel JW, Lubas B, Szczepkowski TW. Proton magnetic relaxation and protein hydration. Nature 1963; 200: 1006-1007.

23 Zimmerman JR, Brittin WE. Nuclear magnetic resonance studies in multiple phase systems: lifetime of a water molecule in an absorbing phase on silica gel. Phys. Chem. 1957; 61: 1328-1333.

24 Grösch L, Noack F. NMR relaxation investigation of water mobility in aqueous bovine serum albumin solutions. Biochim. Biophys. Acta 1976; 453(1): 218-232.

25 Tanttu JI, Sepponen RE, Sipponen JT, Heiskanen J. Tissue parameters $T_{1}, T_{2}, T_{1 \text { rho }}$ and $T_{1 \text { rho }}$ dispersion measured at $0.02 \mathrm{~T}$. Proceedings of the 5th Annual Meeting of the Society of Magnetic Resonance in Medicine, Montreal, QC, Canada, 1986; 1137-1138.

26 Sepponen RE, Tanttu Jl, Suramo I. $\mathrm{T}_{1 \text { rho }}$ and $T_{1 \text { rho }}$ dispersion imaging in patients with cerebral infarction. Proceedings of the 5th Annual Meeting of the Society of Magnetic Resonance in Medicine, Montreal, QC, Canada, 1986; 639-640.

27 Sepponen RE, Tanttu J, Foust RJ, Kinnunen E. T1rho dispersion imaging in multiple sclerosis at $0.1 \mathrm{~T}$. Proceedings of the 9th Annual Meeting of the Society of Magnetic Resonance in Medicine, New York, USA, 1990; 625.

28 Virta $A$, Komu $M$, Kormano $M$. $\mathrm{T}_{1 \text { rho }}$ of protein solutions at very low fields: dependence on molecular weight, concentration, and structure. Magn. Reson. Med. 1997; 37(1): 53-57.

29 Wang N, Xia Y. Anisotropic analysis of multi-component $T_{2}$ and $T_{1 \rho}$ relaxations in achilles tendon by NMR spectroscopy and microscopic MRI. J. Magn. Reson. Imaging 2013; 38(3): 625-633.

30 Chopra S, McClung RED, Jordan RB. Rotating-frame relaxation rates of solvent molecules in solutions of paramagnetic ions undergoing solvent exchange. J. Magn. Reson. 1984; 59(3): 361-372.

31 Ulmer JL, Mathews VP, Hamilton CA, Elster AD, Moran PR. Magnetization transfer or spin-lock? An investigation of off-resonance saturation pulse imaging with varying frequency offsets. Am. J. Neuroradiol. 1996; 17(5): 805-819.

32 Brown RD, Koenig SH. $1 / T_{1 \text { rho }}$ and low-field $1 / T_{1}$ of tissue water protons arise from magnetization transfer to macromolecular solidstate broadened lines. Magn. Reson. Med. 1992; 28(1): 145-152.

33 Markkola AT, Aronen HJ, Paavonen T, Hopsu E, Sipilä LM, Tanttu Jl, Sepponen RE. Spin lock and magnetization transfer imaging of head and neck tumors. Radiology 1996; 200(2): 369-375.

34 Markkola AT, Aronen HJ, Paavonen T, Hopsu E, Sipilä LM, Tanttu Jl, Sepponen RE. T1 rho dispersion imaging of head and neck tumors: a comparison to spin lock and magnetization transfer techniques. J. Magn. Reson. Imaging 1997; 7(5): 873-879.

35 Ramadan UA, Tanttu Jl, Häkkinen AM, Markkola AT, Savolainen SE, Aronen HJ. Simultaneous use of Gd-DTPA and spin-lock imaging: a phantom study. Acad. Radiol. 1998; 5(S1): S141-S144.

36 Yuan J, Zhao F, Ahuja A, Wang YJ. MR chemical exchange imaging with spin-lock technique (CESL): a theoretical analysis of the $z$ spectrum using a two-pool $R_{1 \rho}$ relaxation model beyond the fastexchange limit. Phys. Med. Biol. 2012; 57(24): 8185-8200. 
37 Kimmich R. NMR Tomography, Diffusometry, Relaxometry. Springe Verlag: Berlin, 1997.

38 Abragam A. The Principles of Nuclear Magnetism. Clarendon Press: Oxford, 1961.

39 Jones G. Spin-lattice relaxation in the rotating frame: weak collision case. Phys. Rev. 1966; 148(1): 332-335.

40 Blicharski J. Nuclear magnetic relaxation in rotating frame. Acta. Phys. Pol. A 1972; 41: 223-236.

41 Mangia S, Liimatainen T, Garwood M, Michaeli S. Rotating frame relaxation during adiabatic pulses vs. conventional spin-lock: simulations and experimental results at 4 T. Magn. Reson. Imaging 2009; 27(8): 1074-1087.

42 Michaeli S, Sorce D, Springer C, Ugurbil K, Garwood M. T $1 p$ MRI contrast in the human brain: modulation of the longitudinal rotating frame relaxation shutter-speed during an adiabatic RF pulse. J. Magn. Reson. 2006; 181(1): 135-147.

43 Abergel D, Palmer AG. On the use of the stochastic Liouville equation in nuclear magnetic resonance: application to $R_{1 \rho}$ relaxation in the presence of exchange. Concepts Magn. Reson. A 2003; 19(2): 134-148.

44 Davis DG, Perlman ME, London RE. Direct measurements of the dissociation-rate constant for inhibitor-enzyme complexes via the $T_{1 \rho}$ and $T_{2}$ (CPMG) methods. J. Magn. Reson. B 1994; 104(3): 266-275.

45 Trott $\mathrm{O}$, Palmer AG. $\mathrm{R}_{1 \rho}$ relaxation outside of the fast-exchange limit. J. Magn. Reson. 2002; 154: 157-160.

46 Michaeli S, Sorce DJ, Idiyatullin D, Ugurbil K, Garwood M. Transverse relaxation in the rotating frame induced by chemical exchange. J. Magn. Reson. 2004; 169(2): 293-299.

47 Sorce DJ, Michaeli S, Garwood M. The time-dependence of exchange-induced relaxation during modulated radiofrequency pulses. J. Magn. Reson. 2006; 179(1): 136-139.

48 Sorce DJ, Michaeli S, Garwood M. Relaxation during adiabatic radiofrequency pulses. Curr. Anal. Chem. 2007; 3(3): 239-251.

49 Solomon I. Rotary spin echoes. Phys. Rev. Lett. 1959; 2: 301-305.

50 Witschey WR, Borthakur A, Elliott MA, Mellon E, Niyogi S, Wallman DJ, Wang $C$, Reddy R. Artifacts in $T_{10}$-weighted imaging: compensation for $B_{1}$ and $B_{0}$ field imperfections. J. Magn. Reson. 2007; 186(1): 75-85.

51 Witschey WR, Borthakur A, Elliott MA, Mellon E, Niyogi S, Wang C, Reddy R. Compensation for spin-lock artifacts using an offresonance rotary echo in $\mathrm{T}_{1 \text { poff }}$-weighted imaging. Magn. Reson. Med. 2007; 57(1): 2-7.

52 Wheaton AJ, Borthakur A, Corbo MT, Moonis G, Melhem E, Reddy R. $T_{2 p}$-weighted contrast in MR images of the human brain. Magn. Reson. Med. 2004; 52(6): 1223-1227.

53 Rommel E, Kimmich R, Körperich H, Kunze C, Gersonde K. T1 rho dispersion imaging and localized $\mathrm{T} 1$ rho dispersion relaxometry: application in vivo to mouse adenocarcinoma. Magn. Reson. Med. 1992; 24(1): 149-157.

54 Kimmich R, Barenz J, Weis J. Spin-lock field cycling imaging relaxometry. J. Magn. Reson. 1995; 117(2): 228-234.

55 Tannús A, Garwood M. Adiabatic pulses. NMR Biomed. 1997; 10(8): 423-434

56 Gröhn HI, Michaeli S, Garwood M, Kauppinen RA, Gröhn OHJ. Quantitative $T_{1 \rho}$ and adiabatic Carr-Purcell $T_{2}$ magnetic resonance imaging of human occipital lobe at 4T. Magn. Reson. Med. 2005; 54(1): 14-19.

57 Michaeli S, Gröhn HI, Gröhn OHJ, Sorce DJ, Kauppinen R, Springer CS, Uğurbil K, Garwood M. Exchange-influenced $T_{2 p}$ contrast in human brain images measured with adiabatic radio frequency pulses. Magn. Reson. Med. 2005; 53(4): 823-829.

58 Liimatainen T, Mangia S, Ling W, Ellermann J, Sorce DJ, Garwood M, Michaeli S. Relaxation dispersion in MRI induced by fictitious magnetic fields. J. Magn. Reson. 2011; 209(2): 269-276.

59 Liimatainen T, Hakkarainen $\mathrm{H}$, Mangia S, Huttunen JMJ, Storino C, Idiyatullin D, Sorce D, Garwood M, Michaeli S. MRI contrasts in high rank rotating frames. Magn. Reson. Med. 2014; 73(1): 254-262.

60 Michaeli S, Sorce D, Garwood M. $T_{2 \rho}$ and $T_{1 \rho}$ adiabatic relaxations and contrasts. Curr. Anal. Chem. 2008; 4(1): 8-25.

61 Borthakur A, Wheaton A, Charagundla SR, Shapiro EM, Regatte RR, Akella SV, Kneeland JB, Reddy R. Three-dimensional $T_{10}$-weighted MRI at 1.5 Tesla. J. Magn. Reson. Imaging 2003; 17(6): 730-736.

62 Li X, Wyatt C, Rivoire J, Han E, Chen W, Schooler J, Liang F, Shet K, Souza R, Majumdar S. Simultaneous acquisition of $T_{1 \mathrm{p}}$ and $T_{2}$ quantification in knee cartilage: repeatability and diurnal variation. J. Magn. Reson. Imaging 2014; 39(5): 1287-1293.
63 Dixon WT, Oshinski JN, Trudeau JD, Arnold BC, Pettigrew RI. Myocardial suppression in vivo by spin locking with composite pulses. Magn. Reson. Med. 1996; 36(1): 90-94.

64 Wassef SN, Wemmie J, Johnson CP, Johnson H, Paulsen JS, Long JD, Magnotta VA. $T_{1 \rho}$ imaging in premanifest Huntington disease reveals changes associated with disease progression. Mov. Disord. 2015; 30(8): 1107-1114.

65 Johnson CP, Follmer RL, Oguz I, Warren LA, Christensen GE, Fiedorowicz JG, Magnotta VA, Wemmie JA. Brain abnormalities in bipolar disorder detected by quantitative $T_{1 \mathrm{p}}$ mapping. Molec. Psychiatr. 2015; 20(1): 201-206.

66 Wang C, Zheng J, Sun J, Wang Y, Xia R, Yin Q, Chen W, Ziqian X, Jichun L, Bing Z, Fabao G. Endogenous contrast $T_{1}$ rho cardiac magnetic resonance for myocardial fibrosis in hypertrophic cardiomyopathy patients. J. Cardiol. 2015; 66(6): 520-526.

67 Duvvuri U, Charagundla SR, Kudchodkar SB, Kaufman JH, Kneeland JB, Rizi R, Leigh JS, Reddy R. Human knee: in vivo $T_{10}$-weighted MR imaging at $1.5 \mathrm{~T}$ - preliminary experience. Radiology 2001; 220(3): 822-826.

68 Wheaton AJ, Borthakur A, Charagundla SR, Reddy R. Pulse sequence for multislice $T_{1 \rho}$-weighted MRI. Magn. Reson. Med. 2004; 51(2): 362-369.

69 Wheaton AJ, Borthakur A, Kneeland JB, Regatte RR, Akella SVS, Reddy R. In vivo quantification of $T_{10}$ using a multislice spin-lock pulse sequence. Magn. Reson. Med. 2004; 52(6): 1453-1458.

70 Wang $N$, Xia Y. Dependencies of multi-component $T_{2}$ and $T_{1 \rho}$ relaxation on the anisotropy of collagen fibrils in bovine nasal cartilage. J. Magn. Reson. 2011; 212(1): 124-132.

71 Kogan F, Singh A, Cai K, Haris M, Hariharan H, Reddy R. Investigation of chemical exchange at intermediate exchange rates using a combination of chemical exchange saturation transfer (CEST) and spin-locking methods (CESTrho). Magn. Reson. Med. 2012; 68(1): 107-119.

72 Gonyea JV, Watts R, Applebee A, Andrews T, Hipko S, Nickerson JP, Thornton L, Filippi CG. In vivo quantitative whole-brain $T_{1}$ rho MRI of multiple sclerosis. J. Magn. Reson. Imaging 2015; 42(6): 1623-1630. DOI:10.1002/jmri.24677.

73 Watts R, Andrews T, Hipko S, Gonyea JV, Filippi CG. In vivo wholebrain $T_{1}$-rho mapping across adulthood: normative values and age dependence. J. Magn. Reson. Imaging 2014; 40(2): 376-382.

74 Cobb JG, Kan JH, Gore JC. $T_{1 \rho}$ mapping of pediatric epiphyseal and articular cartilage in the knee. J. Magn. Reson. Imaging 2013; 38(2): 299-305.

75 Borthakur A, Hulvershorn J, Gualtieri E, Wheaton AJ, Charagundla SR, Elliott MA, Reddy R. A pulse sequence for rapid in vivo spinlocked MRI. J. Magn. Reson. Imaging 2006; 23(4): 591-596.

76 Lu J, Dai G, Egi Y, Huang S, Kwon SJ, Lo EH, Kim YR. Characterization of cerebrovascular responses to hyperoxia and hypercapnia using MRI in rat. Neuroimage 2009; 45(4): 1126-1134.

77 Jin T, Autio J, Obata T, Kim SG. Spin-locking versus chemical exchange saturation transfer MRI for investigating chemical exchange process between water and labile metabolite protons. Magn. Reson. Med. 2011; 65(5): 1448-1460.

78 Witschey W, Borthakur A, Elliott MA, Fenty M, Sochor MA, Wang C, Reddy R. $T_{1 \rho}$-prepared balanced gradient echo for rapid 3D $T_{1 \rho}$ MRI. J. Magn. Reson. Imaging 2008; 28(3): 744-754.

79 Paul D, Markl M, Fautz HP, Hennig J. T2-weighted balanced SSFP imaging (T2-TIDE) using variable flip angles. Magn. Reson. Med. 2006; 56(1): 82-93.

80 Witschey WR, Borthakur A, Elliott MA, Magland J, McArdle EL, Wheaton A, Reddy R. Spin-locked balanced steady-state freeprecession (sISSFP). Magn. Reson. Med. 2009; 62(4): 993-1001.

81 Yuan J, Zhao F, Griffith JF, Chan Q, Wang YJ. Optimized efficient liver $T_{1 \rho}$ mapping using limited spin lock times. Phys. Med. Biol. 2012; 57: $1631-1640$.

82 Yuan J, Li Y, Zhao F, Chan Q, Ahuja AT, Wang YJ. Quantification of $T_{1}$ relaxation by using rotary echo spin-lock pulses in the presence of $B_{0}$ inhomogeneity. Phys. Med. Biol. 2012; 57(15): 5003-5016.

83 Mangia S, Carpenter AF, Tyan AE, Eberly LE, Garwood M, Michaeli S. Magnetization transfer and adiabatic $T_{1 \rho}$ MRI reveal abnormalities in normal appearing white matter of subjects with multiple sclerosis. Mult. Scler. 2014; 20(8): 1066-1073.

84 Du J, Statum S, Znamirowski R, Bydder GM, Chung CB. Ultrashort TE $T_{1 \rho}$ magic angle imaging. Magn. Reson. Med. 2013; 69(3): 682-687.

85 Du J, Carl M, Bae WC, Statum S, Chang EY, Bydder GM, Chung CB. Dual inversion recovery ultrashort echo time (DIR-UTE) imaging 
and quantification of the zone of calcified cartilage (ZCC). Osteoarthr. Cartil. 2013; 21(1): 77-85.

86 Borthakur A, Wheaton AJ, Gougoutas AJ, Akella SVS, Regatte RR, Charagundla SR, Reddy R. In vivo measurement of $T_{1 \rho}$ dispersion in the human brain at 1.5 tesla. J. Magn. Reson. Imaging 2004; 19 (4): 403-409.

87 Haris M, McArdle E, Fenty M, Singh A, Davatzikos C, Trojanowski JQ, Melhem ER, Clark CM, Borthakur A. Early marker for Alzheimer's disease: hippocampus T1rho $\left(T_{1 \rho}\right)$ estimation. J. Magn. Reson. Imaging 2009; 29(5): 1008-1012.

88 Haris M, Singh A, Cai K, Davatzikos C, Trojanowski JQ, Melhem ER, Clark C, Borthakur A. T1rho $\left(T_{1 \rho}\right)$ MR imaging in Alzheimer's disease and Parkinson's disease with and without dementia. J. Neurol. 2011; 258(3): 380-385.

89 Markkola AT, Aronen HJ, Ramadan UA, Halavaara JT, Tanttu Jl, Sepponen RE. Determination of $T_{1 \rho}$ values for head and neck tissues at $0.1 \mathrm{~T}$ : a comparison to $T_{1}$ and $T_{2}$ relaxation times. Magn. Reson. Imaging 1998; 16(4): 377-383.

90 Koskinen SK, Niemi PT, Kajander SA, Komu ME. T1rho dispersion profile of rat tissues in vitro at very low locking fields. Magn. Reson. Imaging 2006; 24(3): 295-299.

91 Nestrasil I, Michaeli S, Liimatainen T, Rydeen CE, Kotz CM, Nixon JP, Hanson T, Tuite PJ. $T_{1 \rho}$ and $T_{2 \rho}$ MRI in the evaluation of Parkinson's disease. J. Neurol. 2010; 257(6): 964-968.

92 Deelchand DK, Henry PG, Marjanska M. Effect of Carr-Purcell refocusing pulse trains on transverse relaxation times of metabolites in rat brain at 9.4 Tesla. Magn. Reson. Med. 2014; 73(1): 13-20. DOI:10.1002/mrm.25088.

93 Mangia S, Liimatainen T, Garwood M, Tkac I, Henry PG, Deelchand D, Michaeli S. Frequency offset dependence of adiabatic rotating frame relaxation rate constants: relevance to MRS investigations of metabolite dynamics in vivo. NMR Biomed. 2011; 24(7): 807-814.

94 Andronesi OC, Bhat H, Reuter M, Mukherjee S, Caravan P, Rosen BR. Whole brain mapping of water pools and molecular dynamics with rotating frame MR relaxation using gradient modulated low-power adiabatic pulses. Neuroimage 2014; 89(1): 92-109.

95 Andronesi OC, Ramadan S, Ratai EM, Jennings D, Mountford CE, Sorensen AG. Spectroscopic imaging with improved gradient modulated constant adiabaticity pulses on high-field clinical scanners. J. Magn. Reson. 2010; 203(2): 283-293.

96 Levitt MH, Freeman R, Frenkiel T. Broad-band heteronuclear decoupling. J. Magn. Reson. 1982; 47(2): 328-330.

97 Jokivarsi KT, Niskanen JP, Michaeli S, Gröhn HI, Garwood M, Kauppinen RA, Gröhn OH. Quantitative assessment of water pools by $T_{1}$ and $T_{2 \rho} \mathrm{MRI}$ in acute cerebral ischemia of the rat. J. Cereb. Blood Flow Metab. 2009; 29(1): 206-216.

98 Sierra A, Michaeli S, Niskanen JP, Valonen PK, Gröhn HI, Ylä-Herttuala S, Garwood M, Gröhn OH. Water spin dynamics during apoptotic cell death in glioma gene therapy probed by $T_{1 \rho}$ and $T_{2 \rho}$. Magn. Reson. Med. 2008; 59(6): 1311-1319.

99 Wang L, Regatte RR. $T_{10}$ MRI of human musculoskeletal system. J. Magn. Reson. Imaging 2015; 41(3): 586-600.

100 Rauscher I, Eibera M, Ganter C, Martirosian P, Safi W, Umgelter A, Rummeny EJ, Holzapfel K. Evaluation of $T_{1 \rho}$ as a potential MR biomarker for liver cirrhosis: comparison of healthy control subjects and patients with liver cirrhosis. Eur. J. Radiol. 2014; 83(6): 900-904.
101 Allkemper T, Sagmeister F, Cicinnati V, Beckebaum S, Kooijman H, Kanthak C, Stehling C, Heindel W. Evaluation of fibrotic liver disease with whole-liver $T_{1 \rho}$ MR imaging: a feasibility study at $1.5 \mathrm{~T}$. Radiology 2014; 271(2): 408-415.

102 Takayama Y, Nishie A, Asayama Y, Ushijima Y, Okamoto D, Fujita N, Morita K, Shirabe K, Kotoh K, Kubo Y, Okuaki T, Honda H. $T_{1 \rho}$ relaxation of the liver: a potential biomarker of liver function. J. Magn. Reson. Imaging 2015; 42(1): 188-195.

103 Han Y, Liimatainen T, Gorman RC, Witschey WR. Assessing myocardial disease using T1 $\rho$ MRI. Curr. Cardiovasc. Imaging Rep. 2014; 7(2): 1-9.

104 Klocke NF, Amendola A, Thedens DR, Williams GN, Luty CM, Martin JA, Pedersen DR. Comparison of $T_{1 \rho}$, dGEMRIC, and quantitative $T_{2}$ MRI in preoperative ACL rupture patients. Acad. Radiol. 2013; 20 (1): 99-107.

105 Theologis AA, Haughom B, Liang F, Zhang Y, Majumdar S, Link TM, $\mathrm{Ma} C \mathrm{~B}$, Li X. Comparison of T1rho relaxation times between ACLreconstructed knees and contralateral uninjured knees. Knee Surg. Sports Traumatol. Arthrosc. 2014; 22(2): 298-307.

106 Wang L, Chang G, Bencardino J, Babb JS, Rokito A, Jazrawi L, Sherman O, Regatte RR. T1rho MRI at $3 \mathrm{~T}$ of menisci in patients with acute anterior cruciate ligament (ACL) injury. J. Magn. Reson. Imaging 2015; 41(2): 544-549.

107 Son M, Goodman SB, Chen W, Hargreaves BA, Gold GE, Levenston ME. Regional variation in $T_{1 \rho}$ and $T_{2}$ times in osteoarthritic human menisci: correlation with mechanical properties and matrix composition. Osteoarthr. Cartil. 2013; 21(6): 796-805.

108 Bae WC, Biswas R, Chen K, Chang EY, Chung CB. UTE MRI of the osteochondral junction. Curr. Radiol. Rep. 2014; 2(2): 35.

109 Nguyen AM, Johannessen W, Yoder JH, Wheaton AJ, Vresilovic EJ, Borthakur A, Elliot DM. Noninvasive quantification of human nucleus pulposus pressure with use of T1 rho-weighted magnetic resonance imaging. J. Bone Joint Surg. 2008; 90(4): 796-802.

110 Wang YXJ, Zhao F, Griffith JF, Mok GSP, Leung JCS, Ahuja AT, Yuan J. $T_{1}$ rho and $T_{2}$ relaxation times for lumbar disc degeneration: an in vivo comparative study at 3.0-Tesla MRI. Eur. Radiol. 2013; 23(1): 228-234.

111 Liu Q, Tawackoli W, Pelled G, Fan Z, Jin N, Natsuaki Y, Bi X, Gart A, Bae $H$, Gazit D, Li D. Detection of low back pain using pH level-dependent imaging of the intervertebral disc using the ratio of $R_{1 \rho}$ dispersion and $-\mathrm{OH}$ chemical exchange saturation transfer (RROC). Magn. Reson. Med. 2014; 73(3): 1196-1205. DOI:10.1002/mrm.25186.

112 Hayter CL, Linklater JM, Man KH. State of the art MR imaging techniques for the foot and ankle. Curr. Radiol. Rep. 2014; 2: 42.

113 Johnson CP, Heo H, Thedens DR, Wemmie JA, Magnotta VA. Rapid acquisition strategy for functional $T_{10}$ mapping of the brain. Magn. Reson. Imaging 2014; 32(9): 1067-1077.

114 Bhave S, Lingala SG, Johnson CP, Magnotta VA, Jacob M. Accelerated whole-brain multi-parameter mapping using blind compressed sensing. Magn. Reson. Med. 2015; 75(3): 1175-1186. DOI:10.1002/mrm.25722.

115 Johnson CP, Thedens DR, Magnotta VA. Precision-guided sampling schedules for efficient $T_{1 \rho}$ mapping. J. Magn. Reson. Imaging 2015; 41(1): 242-250.

116 Hectors SJCG, Moonen RPM, Strijkers GJ, Nicolay K. $T_{1 \mathrm{p}}$ mapping for the evaluation of high intensity focused ultrasound tumor treatment. Magn. Reson. Med. 2015; 73(4): 1593-1601. 